\title{
Semi-classical analysis of the Laplace operator with Robin boundary conditions
}

\author{
Rupert L. Frank • Leander Geisinger
}

Received: 11 August 2012 / Accepted: 24 August 2012 / Published online: 5 October 2012

(C) The Author(s) 2012. This article is published with open access at SpringerLink.com

\begin{abstract}
We prove a two-term asymptotic expansion of eigenvalue sums of the Laplacian on a bounded domain with Neumann, or more generally, Robin boundary conditions. We formulate and prove the asymptotics in terms of semi-classical analysis. In this reformulation it is natural to allow the function describing the boundary conditions to depend on the semi-classical parameter and we identify and analyze three different regimes for this dependence.
\end{abstract}

\section{Introduction and main result}

\subsection{Introduction}

The Laplace operator on a bounded domain $\Omega \subset \mathbb{R}^{d}, d \geq 2$, initially defined as a symmetric operator in $L^{2}(\Omega)$ with domain $C_{0}^{\infty}(\Omega)$, admits various self-adjoint extensions that correspond to different boundary conditions. Our goal in this paper is to study how different boundary conditions influence the asymptotic behavior of the eigenvalues.

We consider self-adjoint extensions that are generated by a quadratic form

$$
\int_{\Omega}|\nabla v|^{2} d x+\int_{\partial \Omega} c(x)|v(x)|^{2} d \sigma(x), \quad v \in H^{1}(\Omega) .
$$

\footnotetext{
Communicated by A. Laptev.
}

The authors wish to thank A. Laptev for stimulating their interest in this problem. U.S. NSF grants PHY-1068285 (R.L.F.) and PHY-1122309 (L.G.) and DFG grant GE 2369/1-1 (L.G.) are acknowledged.

R. L. Frank $(\varangle) \cdot$ L. Geisinger

Department of Mathematics, Princeton University, Princeton, NJ 08544, USA

e-mail: rlfrank@math.princeton.edu

L. Geisinger

e-mail: leander@princeton.edu 
Here the form domain $H^{1}(\Omega)$ is the Sobolev space of order $1, d \sigma$ denotes the $d-1$ dimensional surface measure on the boundary $\partial \Omega$, and $c$ is a bounded, real valued function on $\partial \Omega$. This quadratic form induces a unique self-adjoint operator $-\Delta_{c}$ in $L^{2}(\Omega)$ and functions from the domain of $-\Delta_{c}$ satisfy, in an appropriate sense, Robin boundary conditions

$$
\frac{\partial v}{\partial n_{x}}(x)=c(x) v(x), \quad x \in \partial \Omega,
$$

where $\frac{\partial}{\partial n_{x}}$ denotes the inner normal derivative. We remark that $c \equiv 0$ corresponds to the important case of Neumann boundary conditions. The Dirichlet Laplacian, generated by the quadratic form $\int_{\Omega}|\nabla v|^{2} d x$ with form domain $H_{0}^{1}(\Omega)$, can be recovered formally by taking the limit $c \rightarrow \infty$.

If the boundary of $\Omega$ is sufficiently regular (e.g., Lipschitz continuous), the spectrum of $-\Delta_{c}$ is purely discrete: It consists of a sequence of eigenvalues $\lambda_{1}<\lambda_{2} \leq \lambda_{3} \leq \ldots$ that accumulate at infinity only. Here we study how the asymptotic distribution of the eigenvalues depends on the boundary condition induced by the function $c$.

It is a classical result that the eigenvalues satisfy

$$
\lambda_{n}=\frac{4 \pi^{2}}{\left(\omega_{d}|\Omega|\right)^{2 / d}} n^{2 / d}+o\left(n^{2 / d}\right) \text { as } n \rightarrow \infty,
$$

where $|\Omega|$ is the volume of $\Omega$ and $\omega_{d}$ denotes the volume of the unit ball in $\mathbb{R}^{d}$. In the case of Dirichlet boundary conditions these asymptotics go back to [15]. They have been generalized in various ways, in particular, to the case of Robin boundary conditions (1.2); see, for instance, the lecture notes [2].

It has been conjectured by Weyl that (1.3) is the beginning of an asymptotic expansion in $n$ and that the second term should depend on the surface area of $\Omega$. Initially, a weaker form of this conjecture has been verified, not for individual eigenvalues, but for smooth functions of the eigenvalues; see, e.g., [11,12]. For instance, [3] computed in the case of boundary conditions (1.2)

$$
\begin{aligned}
\sum_{j=1}^{\infty} e^{-t \lambda_{j}}= & (4 \pi t)^{-d / 2}\left(|\Omega|+\frac{\sqrt{\pi}}{2}|\partial \Omega| t^{1 / 2}+\frac{1}{3} \int_{\partial \Omega}(H(x)-6 c(x)) d \sigma(x) t+O\left(t^{3 / 2}\right)\right) \\
& \text { as } t \rightarrow 0 .
\end{aligned}
$$

Here $H(x)$ is the mean curvature (the trace of the second fundamental form) at $x \in \partial \Omega$. We see that the second term indeed depends on the surface area $|\partial \Omega|$ and is independent of $c$. The boundary condition enters only in the third order term. (For Dirichlet conditions, however, the sign of the second term flips.) In contrast to (1.3), the expansion (1.4) requires the boundary to be smooth.

A two-term asymptotic formula for individual eigenvalues was eventually shown in a celebrated work of V. Ivrii; see [7-9,14]. He showed that, under a certain condition on the global geometry of $\Omega$ (and some smoothness conditions), one has for boundary 
conditions (1.2)

$$
\lambda_{n}=\frac{4 \pi^{2}}{\left(\omega_{d}|\Omega|\right)^{2 / d}} n^{2 / d}-\frac{2 \pi^{2}}{d} \frac{\omega_{d-1}|\partial \Omega|}{\left(\omega_{d}|\Omega|\right)^{1+1 / d}} n^{1 / d}+o\left(n^{1 / d}\right) \text { as } n \rightarrow \infty .
$$

Again, for any bounded function $c$ the result is the same as for Neumann conditions. We emphasize that (1.5) implies the two-term analogue of (1.4), but not vice versa.

In this paper we shall study an eigenvalue quantity which is intermediate between (1.4) and (1.5), namely, partial sums $\sum_{j=1}^{n} \lambda_{j}$ as $n \rightarrow \infty$ or, equivalently, $\sum_{j=1}^{\infty}$ $\left(\lambda_{j}-\mu\right)_{-}$as $\mu \rightarrow \infty$. These partial sums describe the energy of non-interacting fermionic particles in $\Omega$ at fixed particle number $n$ or at fixed chemical potential $\mu$, respectively. They play an important role in physical applications.

Since the function $\lambda \mapsto(\lambda-\mu)_{-}$is not smooth, we cannot expect that a threeterm asymptotic expansion exists for these eigenvalue sums. Hence, to see the effect of boundary conditions already in the second term of the asymptotic expansion we have to choose energy-dependent boundary conditions. Let us state this problem in a semi-classical set-up. For a small parameter $h>0$ we define self-adjoint operators $H(b)=-h^{2} \Delta_{b / h}-1$ in $L^{2}(\Omega)$ generated by the quadratic form

$$
q_{b}[v]=h^{2} \int_{\Omega}|\nabla v|^{2} d x+h \int_{\partial \Omega} b(x)|v(x)|^{2} d \sigma(x)-\int_{\Omega}|v(x)|^{2} d x
$$

with form domain $H^{1}(\Omega)$. Here $b$ is a bounded function on $\partial \Omega$ that may also depend on $h$. The quadratic form $q_{b}$ induces, in an appropriate sense, $h$-dependent boundary conditions

$$
h \frac{\partial v}{\partial n_{x}}(x)=b(x) v(x), \quad x \in \partial \Omega .
$$

In this introduction, we denote by $E_{n}(b, h)$ the eigenvalues of the operator $-h^{2} \Delta_{b / h}$; consequently, the eigenvalues of $H(b)$ are given by $E_{n}(b, h)-1$. As we explained, our main goal will be to study the sum of the negative eigenvalues of $H(b)$,

$$
\operatorname{Tr} H(b)_{-}=\sum_{n \in \mathbb{N}}\left(E_{n}(b, h)-1\right)_{-}
$$

in the semiclassical limit $h \downarrow 0$. We prove two-term asymptotics and show how the second term depends on the function $b$. Our analysis will show that the asymptotics has different forms in three different regimes depending on the size of $b$ as $h \downarrow 0$. The three different regimes are where $b \rightarrow 0$ as $h \downarrow 0, b$ of order one as $h \downarrow 0$ and $|b| \rightarrow \infty$ as $h \downarrow 0$.

As an example of the first regime, let us consider the case $b=h c$ with a bounded function $c$ independent of $h$. This corresponds to the classical situation discussed 
above, where the boundary condition (1.7) is independent of $h$ and therefore the eigenvalues $E_{n}(b, h)=h^{2} \lambda_{n}$ depend trivially on $h$. Then (1.5) implies

$$
\frac{1}{n} \sum_{j=1}^{n} \lambda_{j}=\frac{4 \pi^{2}}{\left(\omega_{d}|\Omega|\right)^{2 / d}} \frac{d}{d+2} n^{2 / d}-\frac{2 \pi^{2}}{d} \frac{\omega_{d-1}|\partial \Omega|}{\left(\omega_{d}|\Omega|\right)^{1+1 / d}} \frac{d}{d+1} n^{1 / d}+o\left(n^{1 / d}\right) \text { as } n \rightarrow \infty
$$

and this is equivalent, by a simple majorization argument, to

$$
\operatorname{Tr} H(b)_{-}=L_{d}^{(1)}|\Omega| h^{-d}+\frac{1}{4} L_{d-1}^{(1)}|\partial \Omega| h^{-d+1}+o\left(h^{-d+1}\right) \quad \text { as } \quad h \downarrow 0
$$

with $L_{d}^{(1)}=\frac{2}{d+2}(2 \pi)^{-d} \omega_{d}$. Of course, we find again that the first two terms of the asymptotics are independent of the boundary condition. As we shall see, this is characteristic for the whole regime where $b \rightarrow 0$ as $h \downarrow 0$. We emphasize that as a byproduct of our analysis we establish (1.9) independently, without using (1.5); see Theorem 1.2. This includes, as a special case, the Neumann Laplacian.

Among the three regimes mentioned above, the technically most interesting one is when $b$ is independent of $h$. In this case the second term of the semi-classical limit of $\operatorname{Tr} H(b)_{-}$does depend on the local behavior of $b(x)$; see Theorem 1.1 below.

Finally, in Theorem 1.3, we consider functions $b$ such that $|b|$ diverges as $h \downarrow 0$. In this case, the form of the asymptotics depends on whether $b$ is negative somewhere or whether $b$ is non-negative. In the first case, the asymptotics are determined by the negative part of $b$ alone. Moreover, if $b$ diverges fast enough, then the boundary term becomes the leading term and diverges faster than the Weyl term. On the other hand, when $b$ is non-negative the order of the second term is preserved but the coefficient may change.

We obtain these results by further extending the approach developed in $[4,5]$, where we treated the Dirichlet Laplacian and the fractional Laplacian on a domain. One virtue of this approach is that it requires only rather weak regularity assumptions on $\partial \Omega$ and $b$. Essentially, a $C^{1}$ assumption on $\partial \Omega$ and on $b$ suffices for a two-term asymptotics.

We now turn to a more precise description of our assumptions and results.

\subsection{Main results}

Let $\Omega \subset \mathbb{R}^{d}, d \geq 2$, be a bounded domain such that the boundary satisfies a uniform $C^{1}$ condition. That is, the local charts of $\partial \Omega$ are differentiable and their derivatives are uniformly continuous and share a common modulus of continuity; see (4.1) for a precise definition. Moreover, we assume that the boundary coefficient $b$ is a continuous, real-valued function on $\partial \Omega$ and we denote a modulus of continuity by $\beta$, i.e.,

$$
|b(x)-b(y)| \leq \beta(|x-y|)
$$

for all $x, y \in \partial \Omega$. We assume that $\beta$ is non-decreasing. 
We remark that the boundary conditions (1.7) for functions in the operator domain of $H(b)$ need not hold in the classical sense under these weak assumptions on the boundary. For $\partial \Omega \in C^{1}$, however, this operator can still be defined by means of the quadratic form $q_{b}$ and the characterization of the operator domain in terms of the form domain gives a weak sense in which (1.7) are valid. This suffices for our proof.

For a constant $b \in \mathbb{R}$ we set

$$
L_{d}^{(2)}(b)= \begin{cases}C_{d}\left(-\frac{\pi}{4}+\int_{0}^{1}\left(1-p^{2}\right)^{(d+1) / 2} \frac{b}{b^{2}+p^{2}} d p\right) & \text { for } b>0, \\ C_{d} \frac{\pi}{4} & \text { for } b=0, \\ C_{d}\left(-\frac{\pi}{4}+\int_{0}^{1}\left(1-p^{2}\right)^{(d+1) / 2} \frac{b}{b^{2}+p^{2}} d p+\pi\left(b^{2}+1\right)^{(d+1) / 2}\right) & \text { for } b<0,\end{cases}
$$

where $C_{d}=4\left|\mathbb{S}^{d-2}\right|(2 \pi)^{-d}\left(d^{2}-1\right)^{-1}$. This expression comes from the explicit diagonalization of a one-dimensional model operator; see Sect. 3. Although it is not obvious from the definition, the function $L_{d}^{(2)}(b)$ is continuously differentiable and non-increasing; see Lemma 3.5 and the remark after Proposition 3.1. In particular, for $b>0$, we have

$$
-\frac{1}{4} L_{d-1}^{(1)}=\lim _{b \rightarrow \infty} L_{d}^{(2)}(b) \leq L_{d}^{(2)}(b) \leq \lim _{b \downarrow 0} L_{d}^{(2)}(b)=L_{d}^{(2)}(0)=\frac{1}{4} L_{d-1}^{(1)}
$$

with $L_{d-1}^{(1)}$ defined after (1.9).

To control error terms we have to introduce a non-decreasing function $\delta$ : $\left[0,\|b\|_{\infty}\right] \rightarrow[0, \infty)$ such that

$$
\delta(\lambda) \geq|\{x \in \partial \Omega: 0<|b(x)|<\lambda\}|
$$

for all $0<\lambda \leq\|b\|_{\infty}$.

Our first main result is the following.

Theorem 1.1 Let $\partial \Omega \in C^{1}$ and assume that $b$ satisfies (1.10) and (1.13) with $\beta(l)=o(1)$ and $\delta(l)=o(1)$ as $l \downarrow 0$. We write

$$
\operatorname{Tr}(H(b))_{-}=L_{d}^{(1)}|\Omega| h^{-d}+\int_{\partial \Omega} L_{d}^{(2)}(b(x)) d \sigma(x) h^{-d+1}+R_{h} .
$$

Then, for an $h$-independent domain $\Omega$, a given $h$-independent upper bound on $\|b\|_{\infty}$ and given $h$-independent $\beta$ and $\delta$, the asymptotics

$$
R_{h}=o\left(h^{-d+1}\right)
$$

holds uniformly in b satisfying these conditions. 
In other words, in this theorem we claim that $R_{h}=o\left(h^{-d+1}\right)$ if $b$ is independent of $h$. Moreover, we claim that these asymptotics are valid even if $b$ depends on $h$, as long as it can be controlled in some uniform way. More precisely, we prove that given $\beta$ and $\delta$ (both non-decreasing and vanishing at zero) and constants $C>0$ and $\varepsilon>0$, there is an $h_{\varepsilon}>0$ such that $\left|R_{h}\right| \leq \varepsilon h^{-d+1}$ for all $0<h \leq h_{\varepsilon}$ and all $b$ satisfying $\|b\|_{\infty} \leq C$, (1.10) and (1.13). Our proof would also allow us to consider $h$-dependent domains $\Omega$, but we do not track the dependence of the constants in terms of $\Omega$ for the sake of simplicity.

Our next result concerns the case where $\|b\|_{\infty} \rightarrow 0$ as $h \downarrow 0$. We will see that the asymptotics are the same as in Theorem 1.1 with $b=0$. We cannot apply Theorem 1.1 , however, since for $b \not \equiv 0$ we cannot choose $\delta$ independent of $h$ such that (1.13) is satisfied and $\delta(\lambda)=o(1)$ as $\lambda \downarrow 0$. Moreover, we can dispense with the assumption that $b$ is continuous.

Theorem 1.2 Let $\partial \Omega \in C^{1}$ and assume that $b=\theta(h) b_{0}$ with $\theta(h)=o(1)$ as $h \downarrow 0$ and with a bounded function $b_{0}$. We write

$$
\operatorname{Tr}(H(b))_{-}=L_{d}^{(1)}|\Omega| h^{-d}+\frac{1}{4} L_{d-1}^{(1)}|\partial \Omega| h^{-d+1}+R_{h} .
$$

Then, for an $h$-independent domain $\Omega$ and a given $h$-independent upper bound on $\left\|b_{0}\right\|_{\infty}$, the asymptotics

$$
R_{h}=o\left(h^{-d+1}\right)
$$

holds uniformly in b satisfying these conditions.

We refer to (2.17) for an explicit bound on $R_{h}$. This holds, in particular, for Neumann boundary conditions.

Our third result concerns the case where $b=\Theta(h) b_{0}$ with $\Theta(h) \rightarrow \infty$.

Theorem 1.3 Let $\partial \Omega \in C^{1}$. Assume $b=\Theta(h) b_{0}$ with $\Theta^{-1}(h)=o(1)$ as $h \downarrow 0$ and with $b_{0}$ satisfying (1.10) with $\beta(l)=o(1)$ as $l \downarrow 0$. We write

$$
\operatorname{Tr}(H(b))_{-}=L_{d}^{(1)}|\Omega| h^{-d}+\pi C_{d} \int_{\partial \Omega} b(x)_{-}^{d+1} d \sigma(x) h^{-d+1}+R_{h} .
$$

Then, for an $h$-independent domain $\Omega$, a given $h$-independent upper bound on $\left\|b_{0}\right\|_{\infty}$ and a given $h$-independent $\beta$, the asymptotics

$$
R_{h}=o\left(\Theta(h)^{d+1} h^{-d+1}\right)
$$

holds uniform in $b$ satisfying these conditions.

If, in addition, $b(x) \geq 0$ for all $x \in \partial \Omega, \delta(\lambda)=o(1)$ as $\lambda \downarrow 0$, and $\beta(M h) \Theta(h)=$ $o$ (1) as $h \downarrow 0$ for every fixed $M>0$, then

$\operatorname{Tr}(H(b))_{-}=L_{d}^{(1)}|\Omega| h^{-d}-\frac{1}{4} L_{d-1}^{(1)}\left|\partial \Omega_{+}\right| h^{-d+1}+\frac{1}{4} L_{d-1}^{(1)}\left|\partial \Omega_{0}\right| h^{-d+1}+o\left(h^{-d+1}\right)$,

where $\partial \Omega_{+}=\{x \in \partial \Omega: b(x)>0\}$ and $\partial \Omega_{0}=\{x \in \partial \Omega: b(x)=0\}$. 
We emphasize that, if the negative part of $b$ does not vanish and $\Theta(h)=h^{\gamma}$ with $\gamma=1 /(d+1)$, then the order of the boundary term is the same as the order of the Weyl term. For $\gamma>1 /(d+1)$ the boundary term becomes the leading term.

Since $\beta(l)$ vanishes at most linearly in $l$ for non-constant $b$, the condition $\beta(M h) \Theta(h)=o(1)$ as $h \downarrow 0$ in the second part of the theorem implies $\Theta(h)=$ $o\left(h^{-1}\right)$. Our techniques do not allow us to consider faster growing $b$ 's and we do not know whether one still can expect the result in that case.

\section{Strategy of the proof}

In this section we outline the main steps of our proof. In particular, we explain how the main results follow from local estimates.

First, we localize the operator $H(b)$ into balls, whose size varies depending on the distance to the complement of $\Omega[6,13]$. Then we analyze the local asymptotics separately in the bulk and close to the boundary.

To localize, let $d(u)=\inf \{|x-u|: x \notin \Omega\}$ denote the distance of $u \in \mathbb{R}^{d}$ to the complement of $\Omega$. We set

$$
l(u)=\frac{1}{2}\left(1+\left(d(u)^{2}+l_{0}^{2}\right)^{-1 / 2}\right)^{-1},
$$

where $0<l_{0} \leq 1$ is a parameter depending only on $h$. Eventually, we will choose $l_{0}=o(1)$ as $h \downarrow 0$. In Sect. 5 we introduce real-valued functions $\phi_{u} \in C_{0}^{\infty}\left(\mathbb{R}^{d}\right)$ with support in $B_{u}=\left\{x \in \mathbb{R}^{d}:|x-u|<l(u)\right\}$. For all $u \in \mathbb{R}^{d}$ these functions satisfy

$$
\left\|\phi_{u}\right\|_{\infty} \leq C, \quad\left\|\nabla \phi_{u}\right\|_{\infty} \leq C l(u)^{-1}
$$

and, for all $x \in \mathbb{R}^{d}$,

$$
\int_{\mathbb{R}^{d}} \phi_{u}^{2}(x) l(u)^{-d} d u=1
$$

Here and in the following the letter $C$ denotes various positive constants that are independent of $u, l_{0}$ and $h$, but may vary from line to line. To estimate error terms in the following results we put

$$
b_{m}=\inf _{x \in \partial \Omega} b(x) .
$$

Proposition 2.1 There is a constant $C_{\Omega}>0$ such that for $0<l_{0} \leq C_{\Omega}^{-1}$ and $0<h \leq l_{0} / 4$ the estimates

$$
-C\left(1+\left(b_{m}\right)_{-}^{d+1} h l_{0}^{-1}\right) l_{0}^{-1} h^{-d+2} \leq \int_{\mathbb{R}^{d}} \operatorname{Tr}\left(\phi_{u} H(b) \phi_{u}\right)_{-} l(u)^{-d} d u-\operatorname{Tr}(H(b))_{-} \leq 0
$$

hold. 
This proposition will be proved in Sect. 5 .

In view of this result one can analyze the asymptotic behavior of $\operatorname{Tr}\left(\phi_{u} H(b) \phi_{u}\right)_{-}$ separately on different parts of $\Omega$. First, we consider the bulk, where the influence of the boundary is not felt.

Proposition 2.2 Let $\phi \in C_{0}^{1}(\Omega)$ be supported in a ball of radius $l>0$ and let

$$
\|\nabla \phi\|_{\infty} \leq C_{\phi} l^{-1}
$$

Then for all $h>0$ the estimates

$$
0 \leq L_{d}^{(1)} \int_{\Omega} \phi^{2}(x) d x h^{-d}-\operatorname{Tr}(\phi H(b) \phi)_{-} \leq C l^{d-2} h^{-d+2}
$$

hold, with a constant $C>0$ depending only on $C_{\phi}$.

For $\phi \in C_{0}^{1}(\Omega)$ we have $\phi H(b) \phi=\phi\left(-h^{2} \Delta-1\right) \phi$, where $-\Delta$ is defined on the whole space $L^{2}\left(\mathbb{R}^{d}\right)$ with form domain $H^{1}\left(\mathbb{R}^{d}\right)$. Hence, this result is independent of the boundary coefficient $b$ and the proof of Proposition 2.2 is the same as in [4].

Close to the boundary of $\Omega$, more precisely, if the support of $\phi$ intersects the boundary, a term of order $h^{-d+1}$ appears that depends on $b$. In this situation let $B$ be a ball containing the support of $\phi$ and put

$$
b^{-}=\inf _{x \in \partial \Omega \cap B} b(x), \quad b^{i}=\inf _{x \in \partial \Omega \cap B}|b(x)|, \quad b^{s}=\sup _{x \in \partial \Omega \cap B}|b(x)| .
$$

To state the remainder estimate we denote by $\omega$ a modulus of continuity of the boundary of $\Omega$; see (4.1) for a precise definition.

Proposition 2.3 Let $\phi \in C_{0}^{1}\left(\mathbb{R}^{d}\right)$ be supported in a ball of radius $l>0$ and let inequalities (1.10) and (2.4) be satisfied. Then there is a constant $C_{\Omega}>0$ such that for $0<l \leq C_{\Omega}^{-1}$ and $0<h \leq l$ we have

$$
\begin{aligned}
\operatorname{Tr}(\phi H(b) \phi)_{-}= & L_{d}^{(1)} \int_{\Omega} \phi^{2}(x) d x h^{-d}+\int_{\partial \Omega} L_{d}^{(2)}(b(x)) \phi^{2}(x) d \sigma(x) h^{-d+1} \\
& +R_{b d}\left(h, l, b^{-}, b^{i}\right)
\end{aligned}
$$

with

$$
\begin{aligned}
&\left|R_{b d}\left(h, l, b^{-}, b^{i}\right)\right| \leq C \frac{l^{d}}{h^{d}}( \frac{h^{2}}{l^{2}}\left(1+\frac{1+\left(b^{-}\right)_{-}^{d+1}}{b^{i}}\right)+\omega(l)\left(1+\frac{h}{l}\left(b^{-}\right)_{-}^{d+1}\right) \\
&\left.+\frac{h}{l}\left(1+\left(b^{-}\right)_{-}^{d}\right) \beta(l)\right) .
\end{aligned}
$$


For $b^{s} \leq h / l$ we also have

$\operatorname{Tr}(\phi H(b) \phi)_{-}=L_{d}^{(1)} \int_{\Omega} \phi^{2}(x) d x h^{-d}+\frac{1}{4} L_{d-1}^{(1)} \int_{\partial \Omega} \phi^{2}(x) d \sigma(x) h^{-d+1}+R_{0}\left(h, l, b^{s}\right)$

with

$$
\left|R_{0}\left(h, l, b^{s}\right)\right| \leq C l^{d} h^{-d}\left(l^{-2} h^{2}+b^{s}\left(1+\left|\ln b^{s}\right|\right)+\omega(l)\right) .
$$

Here the constants $C>0$ depend only on $\Omega$ and $C_{\phi}$.

The first statement in Proposition 2.3 is the crucial result of this section. It yields a precise estimate with the boundary term including the correct constant $L_{d}^{(2)}(b)$. However, we obtain an error term that diverges as $b^{i} \rightarrow 0$. To overcome this effect we also need the second statement for $b$ very close to zero. The next lemma is a simplified version of (2.8), where we estimate the boundary term by $C l^{d-1} h^{-d+1}$.

Lemma 2.4 Under the conditions of Proposition 2.3 there is a constant $C_{\Omega}>0$ such that for $0<l \leq C_{\Omega}^{-1}$ and $0<h \leq l$ we have

$$
\operatorname{Tr}(\phi H(b) \phi)_{-}=L_{d}^{(1)} \int_{\Omega} \phi^{2}(x) d x h^{-d}+R_{0}^{\prime}\left(h, l, b^{-}\right)
$$

with

$\left|R_{0}^{\prime}\left(h, l, b^{-}\right)\right| \leq C l^{d} h^{-d}\left(l^{-1} h+\omega(l)+l^{-1} h\left(b^{-}\right)_{-}^{d+1}\left(\min \left\{l h^{-1}\left(b^{-}\right)_{-}, 1\right\}+\omega(l)\right)\right)$.

Both Proposition 2.3 and Lemma 2.4 will be proved in Sect. 4.

Based on the preceding results we can now give the proofs of our main results.

Proof of Theorem 1.1 We fix two parameters $0<\lambda \leq 1$ and $0<\mu \leq 1 / 4$ and set $l_{0}=h \mu^{-1}$. Let us recall the definition of $l(u)$ from $(2.1)$ and of $B_{u}=\left\{x \in \mathbb{R}^{d}\right.$ : $|x-u|<l(u)\}$. We set

$$
U=\left\{u \in \mathbb{R}^{d}: \partial \Omega \cap B_{u} \neq \emptyset\right\}
$$

First, we need to estimate $l(u)$ uniformly. Note that by definition

$$
l(u) \geq \frac{1}{4} \min (d(u), 1) \text { and } l(u) \geq \frac{l_{0}}{4} \geq h
$$

for all $u \in \mathbb{R}^{d}$. Moreover, for $u \in U$, we have $d(u) \leq l(u)$ and

$$
l(u) \leq l_{0} / \sqrt{3}=h /(\sqrt{3} \mu) .
$$


For $0<h \leq \mu C_{\Omega}^{-1}$ it follows that $l_{0} \leq C_{\Omega}^{-1}$ and $l(u) \leq C_{\Omega}^{-1}$ for all $u \in U$. Moreover, $h=\mu l_{0} \leq l_{0} / 4 \leq l(u)$. Therefore the assumptions of Proposition 2.1, Proposition 2.2, and Proposition 2.3 are satisfied.

Depending on $\lambda$ we decompose $U$ into the regions

$$
\begin{aligned}
U_{0} & =\left\{u \in U: \exists x \in \partial \Omega \cap B_{u}: b(x)=0\right\}, \\
U^{*} & =\left\{u \in U: \forall x \in \partial \Omega \cap B_{u}: 0<|b(x)|<\lambda\right\}, \\
U_{>} & =\left\{u \in U: \exists x \in \partial \Omega \cap B_{u}:|b(x)| \geq \lambda\right\} .
\end{aligned}
$$

We remark that $U=U_{0} \cup U^{*} \cup U_{>}$and that the three sets are mutually disjoint. Indeed, if $x \in \partial \Omega \cap B_{u}$ with $u \in U_{0}$, then by the continuity of $b$, see (1.10),

$$
|b(x)| \leq \beta(l(u)) \leq \beta\left(\frac{h}{\sqrt{3} \mu}\right),
$$

and similarly, if $x \in \partial \Omega \cap B_{u}$ with $u \in U_{>}$,

$$
|b(x)| \geq \lambda-\beta\left(\frac{h}{\sqrt{3} \mu}\right) .
$$

Thus, by our assumption on $\beta$, we have for all sufficiently small $h>0$ (depending on $\mu$ and $\lambda$ ) that $\beta\left(\frac{h}{\sqrt{3} \mu}\right)<\lambda-\beta\left(\frac{h}{\sqrt{3} \mu}\right)$. Thus $U_{0} \cap U_{>}=\emptyset$, as claimed. We can also make sure that for all sufficiently small $h$

$$
|b(x)| \leq \sqrt{3} \mu \leq h / l(u) \quad \text { for all } x \in \partial \Omega \cap B_{u} \text { with } u \in U_{0}
$$

and

$$
|b(x)| \geq \lambda / 2 \quad \text { for all } x \in \partial \Omega \cap B_{u} \text { with } u \in U_{>} .
$$

To estimate error terms we put, similarly as in (2.6),

$$
b_{u}^{-}=\inf _{x \in \partial \Omega \cap B_{u}} b(x), \quad b_{u}^{i}=\inf _{x \in \partial \Omega \cap B_{u}}|b(x)|, \quad b_{u}^{s}=\sup _{x \in \partial \Omega \cap B_{u}}|b(x)| .
$$

First, we apply Proposition 2.1. Then, in order to estimate $\operatorname{Tr}\left(\phi_{u} H(b) \phi_{u}\right)_{-}$, we use (2.5) for $u \in \Omega \backslash U$, (2.7) for $u \in U_{>}$, (2.8) for $u \in U_{0}$, and (2.9) for $u \in U^{*}$. We obtain

$$
\begin{aligned}
-R^{-} \leq & L_{d}^{(1)} \int_{\mathbb{R}^{d}} \int_{\Omega} \phi_{u}^{2}(x) \frac{d x d u}{l(u)^{d} h^{d}}+\int_{U} \int_{\partial \Omega} L_{d}^{(2)}(b(x)) \phi_{u}^{2}(x) \frac{d \sigma(x) d u}{l(u)^{d} h^{d-1}} \\
& -\operatorname{Tr}(H(b))_{-} \leq R^{+}
\end{aligned}
$$


with

$$
\begin{aligned}
R^{-}= & \int_{U_{>}}\left|R_{b d}\left(h, l(u), b_{u}^{-}, b_{u}^{i}\right)\right| \frac{d u}{l(u)^{d}}+\int_{U_{0}}\left|R_{0}\left(h, l(u), b_{u}^{s}\right)\right| \frac{d u}{l(u)^{d}} \\
& +\int_{U_{0}} \int_{\partial \Omega}\left|L_{d}^{(2)}(b(x))-\frac{1}{4} L_{d-1}^{(1)}\right| \phi_{u}^{2}(x) \frac{d \sigma(x) d u}{l(u)^{d} h^{d-1}}+\int_{U^{*}}\left|R_{0}^{\prime}\left(h, l(u), b_{u}^{-}\right)\right| \frac{d u}{l(u)^{d}} \\
& +\int_{U^{*} \partial \Omega}\left|L_{d}^{(2)}(b(x))\right| \phi_{u}^{2}(x) \frac{d \sigma(x) d u}{l(u)^{d} h^{d-1}}+C l_{0}^{-1} h^{-d+2}\left(1+\left(b_{m}\right)_{-}^{d+1} h l_{0}^{-1}\right)
\end{aligned}
$$

and

$$
\begin{aligned}
R^{+}= & \int_{U_{>}}\left|R_{b d}\left(h, l(u), b_{u}^{-}, b_{u}^{i}\right)\right| \frac{d u}{l(u)^{d}}+\int_{U_{0}}\left|R_{0}\left(h, l(u), b_{u}^{s}\right)\right| \frac{d u}{l(u)^{d}} \\
& +\int_{U_{0}} \int_{\partial \Omega}\left|L_{d}^{(2)}(b(x))-\frac{1}{4} L_{d-1}^{(1)}\right| \phi_{u}^{2}(x) \frac{d \sigma(x) d u}{l(u)^{d} h^{d-1}}+\int_{U^{*}}\left|R_{0}^{\prime}\left(h, l(u), b_{u}^{-}\right)\right| \frac{d u}{l(u)^{d}} \\
& +\int_{U^{*} \partial \Omega}\left|L_{d}^{(2)}(b(x))\right| \phi_{u}^{2}(x) \frac{d \sigma(x) d u}{l(u)^{d} h^{d-1}}+C \int_{\Omega \backslash U} l(u)^{-2} d u h^{-d+2} .
\end{aligned}
$$

In the main term we change the order of integration and use the partition of unity property (2.3) to obtain

$$
L_{d}^{(1)} \int_{\mathbb{R}^{d}} \int_{\Omega} \phi_{u}^{2}(x) d x \frac{d u}{l(u)^{d}} h^{-d}=L_{d}^{(1)}|\Omega| h^{-d}
$$

and

$$
\int_{U} \int_{\partial \Omega} L_{d}^{(2)}(b(x)) \phi_{u}^{2}(x) \frac{d \sigma(x) d u}{l(u)^{d} h^{d-1}}=\int_{\partial \Omega} L_{d}^{(2)}(b(x)) d \sigma(x) h^{-d+1} .
$$

Thus, we get

$$
-R^{-} \leq L_{d}^{(1)}|\Omega| h^{-d}+\int_{\partial \Omega} L_{d}^{(2)}(b(x)) d \sigma(x) h^{-d+1}-\operatorname{Tr}(H(b))_{-} \leq R^{+},
$$

and to complete the proof it remains to bound the remainder terms $R^{ \pm}$.

We now argue that the last term in the definition of $R^{+}$is controlled by the last term in the definition of $R^{-}$, that is, by

$$
C l_{0}^{-1} h^{-d+2}\left(1+\left(b_{m}\right)_{-}^{d+1} h l_{0}^{-1}\right) \leq C h^{-d+1} \mu\left(1+\|b\|_{\infty}^{d+1}\right) .
$$


To prove this, we note that for $u \in \Omega \backslash U$ we have $d(u) \geq l(u) \geq l_{0} / 4$ and

$$
\int_{\Omega \backslash U} l(u)^{-2} d u \leq C\left(1+\int_{\left\{d(u) \geq l_{0} / 4\right\}} d(u)^{-2} d u\right) \leq C\left(1+\int_{l_{0} / 4}^{\infty} t^{-2}\left|\partial \Omega_{t}\right| d t\right) .
$$

Here $\left|\partial \Omega_{t}\right|$ denotes the surface area of the boundary of $\Omega_{t}=\{x \in \Omega: d(x)>t\}$. Using the fact that $\left|\partial \Omega_{t}\right|$ is uniformly bounded and that $\left|\partial \Omega_{t}\right|=0$ for large $t$, we get

$$
\int_{\Omega \backslash U} l(u)^{-2} d u \leq C l_{0}^{-1} \leq C \mu h^{-1} .
$$

This proves that the last term in $R^{+}$is bounded by (2.14).

To proceed, we note that inequalities (2.11) and (2.10) show that $l(u)$ for $u \in U$ is comparable with $l_{0}$. Since $B_{u} \cap \partial \Omega \neq \emptyset$ we find $d(u)<l(u) \leq C l_{0}$ and, for any positive and non-decreasing function $r$,

$$
\int_{U} r(l(u)) d u \leq C r\left(C l_{0}\right) \int_{\left\{d(u) \leq l_{0}\right\}} d u \leq C r\left(C l_{0}\right) l_{0} .
$$

Thus, if we insert the identity $l_{0}=h \mu^{-1}$ and the estimates (2.12), (2.13), (2.16) and (2.15) into the expressions for $R^{-}$and $R^{+}$, we find that both are bounded by a constant times

$$
\begin{aligned}
R= & h^{-d+1}\left(1+\|b\|_{\infty}^{d+1}\right)\left(\mu+\frac{\mu}{\lambda}+\omega\left(\frac{C h}{\mu}\right) \frac{1}{\mu}+\beta\left(\frac{C h}{\mu}\right)\right) \\
& +h^{-d+1}\left(\left|U^{*}\right| \frac{\mu}{h}\left(1+\omega\left(\frac{C h}{\mu}\right) \frac{1}{\mu}\right)+\frac{1}{\mu} \beta\left(\frac{h}{\sqrt{3} \mu}\right)\left(1+\left|\ln \beta\left(\frac{h}{\sqrt{3} \mu}\right)\right|\right)\right) .
\end{aligned}
$$

Here we used the facts that $\left|U_{0}\right| \leq|U| \leq C l_{0}$ and $\left|L_{d}^{(2)}(b(x))-\frac{1}{4} L_{d-1}^{(1)}\right| \leq$ $C \beta(h / \sqrt{3} \mu)$ for $x \in B_{u} \cap \partial \Omega$ with $u \in U_{0}$.

To estimate $\left|U^{*}\right|$ we apply Lemma 6.1, given in Appendix A, to the set $N=$ $\{x \in \partial \Omega: 0<|b(x)|<\lambda\}$. By the defining property (1.13) of $\delta$ we obtain

$$
\underset{h \downarrow 0}{\limsup } \frac{\mu}{h}\left|U^{*}\right|=\limsup _{l_{0} \downarrow 0} \frac{1}{l_{0}}\left|U^{*}\right| \leq C \delta(\lambda) .
$$

Hence, by our assumptions on $\omega$ and $\beta$, it follows that

$$
\underset{h \downarrow 0}{\limsup }\left(h^{d-1} R\right) \leq\left(1+\|b\|_{\infty}^{d+1}\right)\left(\mu+\frac{\mu}{\lambda}\right)+C \delta(\lambda) .
$$

By our assumption on $\delta$, the right hand side can be made arbitrarily small by choosing first $\lambda$ small and then $\mu$ small. This completes the proof of Theorem 1.1. 
Proof of Theorem 1.2 This proof is similar to the proof of Theorem 1.1 above. Again we choose

$$
U=\left\{u \in \mathbb{R}^{d}: \partial \Omega \cap B_{u} \neq \emptyset\right\}
$$

and we assume that $l_{0}=h \mu^{-1}$ with $0<\mu \leq 1 / 4$. Then $h \leq l(u)$ for all $u \in U$.

Let us choose $h$ small enough such that $|b(x)|=\left|b_{0}(x)\right| \theta(h) \leq \sqrt{3} \mu \leq h / l(u)$ for all $x \in \partial \Omega$ and $u \in U$. Then we can apply (2.8) to estimate $\operatorname{Tr}\left(\phi_{u} H(b) \phi_{u}\right)_{-}$for $u \in U$. This yields

$$
\begin{aligned}
\left|\operatorname{Tr}(H(b))_{-}-L_{d}^{(1)}\right| \Omega\left|h^{-d}-\frac{1}{4} L_{d-1}^{(1)}\right| \partial \Omega\left|h^{-d+1}\right| \leq & \int_{U}\left|R_{0}\left(h, l(u), b_{u}^{s}\right)\right| \frac{d u}{l(u)^{d}} \\
& +C l_{0}^{-1} h^{-d+2} .
\end{aligned}
$$

Similarly as above we bound

$$
\int_{U}\left|R_{0}\left(h, l(u), b_{u}^{s}\right)\right| \frac{d u}{l(u)^{d}} \leq C h^{-d+1}\left(\mu+\omega\left(\frac{C h}{\mu}\right) \frac{1}{\mu}+\|b\|_{\infty}\left(1+\left|\ln \|b\|_{\infty}\right|\right) \frac{1}{\mu}\right) .
$$

We multiply this by $h^{d+1}$ and let $h \downarrow 0$ recalling that $\|b\|_{\infty}=\theta(h)\left\|b_{0}\right\|_{\infty}=o(1)$. Since $\mu$ can be chosen arbitrarily small, we obtain the claimed asymptotics.

In this case the proof shows that the remainder $R_{h}$ from Theorem 1.2 can be estimated as follows. For all $0<\mu \leq 1 / 4$ we have

$$
\left|R_{h}\right| \leq C h^{-d+1}\left(\mu+\omega\left(\frac{C h}{\mu}\right) \frac{1}{\mu}+\theta(h)\left\|b_{0}\right\|_{\infty}\left(1+\left|\ln \left(\theta(h)\left\|b_{0}\right\|_{\infty}\right)\right|\right) \frac{1}{\mu}\right) .
$$

Proof of Theorem 1.3 First, we assume that the negative part of $b$ does not vanish. Then in the same way as in the proof of Theorem 1.1 we fix parameters $0<\lambda \leq 1$ and $0<\mu \leq 1 / 4$ and set $l_{0}=h \mu^{-1}$ and

$$
U=\left\{u \in \mathbb{R}^{d}: \partial \Omega \cap B_{u} \neq \emptyset\right\} .
$$

Here we choose

$$
\tilde{U}^{*}=\left\{u \in U: \exists x \in \partial \Omega \cap B_{u}:|b(x)|<\lambda\right\}
$$

Then, similar as in the proof of Theorem 1.1, by applying (2.5) for $u \in \Omega \backslash U$, (2.7) for $u \in U \backslash \tilde{U}^{*}$, and (2.9) for $u \in \tilde{U}^{*}$, we obtain

$$
\left|\operatorname{Tr}(H(b))_{-}-L_{d}^{(1)}\right| \Omega\left|h^{-d}-\int_{\partial \Omega} L_{d}^{(2)}(b(x)) d x h^{-d+1}\right| \leq C R
$$


with

$$
\begin{aligned}
R= & h^{-d+1}\left(1+\|b\|_{\infty}^{d+1}\right)\left(\mu+\frac{\mu}{\lambda}+\omega\left(\frac{C h}{\mu}\right) \frac{1}{\mu}+\frac{\Theta(h)}{1+\|b\|_{\infty}} \beta\left(\frac{C h}{\mu}\right)\right) \\
& +h^{-d+1}\left(1+\Theta(h) \beta\left(\frac{C h}{\mu}\right)\right)^{d+1}\left(1+\omega\left(\frac{C h}{\mu}\right)\right) .
\end{aligned}
$$

We emphasize that in order to arrive at this bound we used the estimates $\left|\tilde{U}^{*}\right| \leq|U| \leq$ $C l_{0}$ and

$$
\left|L_{d}^{(2)}(b(x))\right| \leq C\left(1+\Theta(h) \beta\left(\frac{h}{\sqrt{3} \mu}\right)\right)^{d+1}
$$

for $x \in \partial \Omega \cap B_{u}$ with $u \in U^{*}$. (Note also that the role of $\beta$ in Proposition 2.3 is now played by $\Theta(h) \beta$.)

To simplify the main term we note that $L_{d}^{(2)}(b)=C_{d} \pi b^{d+1}+O\left(\Theta(h)^{d-1}\right)$ as $h \downarrow 0$. Hence,

$$
\int_{\partial \Omega} L_{d}^{(2)}(b(x)) d x=C_{d} \pi \int_{\partial \Omega} b(x)_{-}^{d+1} d \sigma(x) h^{-d+1}+O\left(\Theta(h)^{d-1} h^{-d+1}\right) .
$$

It remains to note that

$$
\limsup _{h \downarrow 0}\left(h^{d-1} \Theta(h)^{-d-1} R\right) \leq C\left(\mu+\frac{\mu}{\lambda}\right)
$$

can be made arbitrarily small. (Since we only assume an $h$-independent upper bound on $\left\|b_{0}\right\|_{\infty}$, one needs to distinguish here the cases whether $\lim \inf \Theta^{-1}\left(1+\|b\|_{\infty}\right)$ is positive or zero.)

We now turn to the proof of the second part of the theorem. If the boundary coefficient $b$ is non-negative we argue in the same way as in the proof of Theorem 1.1. We obtain

$$
\begin{gathered}
\left|\operatorname{Tr}(H(b))_{-}-L_{d}^{(1)}\right| \Omega\left|h^{-d}-\int_{\partial \Omega} L_{d}^{(2)}(b(x)) d \sigma(x) h^{-d+1}\right| \\
\leq C h^{-d+1}\left(\mu+\frac{\mu}{\lambda}+\omega\left(\frac{C h}{\mu}\right) \frac{1}{\mu}+\Theta(h) \beta\left(\frac{C h}{\mu}\right)+\left|U^{*}\right| \frac{\mu}{h}\left(1+\omega\left(\frac{C h}{\mu}\right) \frac{1}{\mu}\right)\right. \\
\left.+\frac{1}{\mu} \Theta(h) \beta\left(\frac{h}{\sqrt{3} \mu}\right)\left(1+\left|\ln \left(\Theta(h) \beta\left(\frac{h}{\sqrt{3} \mu}\right)\right)\right|\right)\right) .
\end{gathered}
$$


In this case the continuity of $L_{d}^{(2)}(b)$, see (1.12), implies

$$
\int_{\partial \Omega} L_{d}^{(2)}(b(x)) d \sigma(x)=-\frac{1}{4} L_{d-1}^{(1)}\left|\partial \Omega_{+}\right|+\frac{1}{4} L_{d-1}^{(1)}\left|\partial \Omega_{0}\right|+o(1),
$$

by dominated convergence as $h \downarrow 0$. Again applying Lemma 6.1 in the same way as in the proof of Theorem 1.1 we see that all terms equal $o\left(h^{-d+1}\right)$ as $h \downarrow 0$.

To summarize this section, we have reduced the proof of our main results to the proof of Proposition 2.1, Proposition 2.3 and Lemma 2.4.

\section{Local asymptotics in the half-space}

From a technical point of view, this section is the heart of our proof. We analyze in great detail a model operator which is explicitly diagonalizable. More precisely, we prove local estimates corresponding to Proposition 2.3 in the case where $\Omega$ is the halfspace $\mathbb{R}_{+}^{d}=\left\{\left(x^{\prime}, x_{d}\right) \in \mathbb{R}^{d-1} \times \mathbb{R}_{+}\right\}$and the boundary coefficient $b$ does not depend on $x$. Let $H^{+}(b)=-h^{2} \Delta-1$ be the self-adjoint operator in $L^{2}\left(\mathbb{R}_{+}^{d}\right)$ generated by the quadratic form

$$
q_{b}^{+}[v]=h^{2} \int_{\mathbb{R}_{+}^{d}}|\nabla v(x)|^{2} d x+h b \int_{\mathbb{R}^{d-1}}\left|v\left(x^{\prime}, 0\right)\right|^{2} d x^{\prime}-\int_{\mathbb{R}_{+}^{d}}|v(x)|^{2} d x
$$

with form domain $H^{1}\left(\mathbb{R}_{+}^{d}\right)$ and with a real constant $b$ independent of $x$.

\subsection{Statement of the results}

Our goal in this section is to prove the following

Proposition 3.1 Assume that $b \in \mathbb{R}$ is constant. Let $\phi \in C_{0}^{1}\left(\mathbb{R}^{d}\right)$ be supported in a ball of radius $l>0$ and let (2.4) be satisfied. Then for $h>0$

$$
\begin{aligned}
\operatorname{Tr}\left(\phi H^{+}(b) \phi\right)_{-}= & L_{d}^{(1)} \int_{\mathbb{R}_{+}^{d}} \phi^{2}(x) d x h^{-d}+L_{d}^{(2)}(b) \int_{\mathbb{R}^{d-1}} \phi^{2}\left(x^{\prime}, 0\right) d x^{\prime} h^{-d+1} \\
& +R_{h s}(h, l, b)
\end{aligned}
$$

with

$$
\left|R_{h s}(h, l, b)\right| \leq C l^{d-2} h^{-d+2}\left(1+\frac{1+b_{-}^{d+1}}{|b|}\right) .
$$


For $|b| \leq h / l \leq 1$ we also have

$$
\begin{aligned}
\operatorname{Tr}\left(\phi H^{+}(b) \phi\right)_{-}= & L_{d}^{(1)} \int_{\mathbb{R}_{+}^{d}} \phi^{2}(x) d x h^{-d}+\frac{1}{4} L_{d-1}^{(1)} \int_{\mathbb{R}^{d-1}} \phi^{2}\left(x^{\prime}, 0\right) d x^{\prime} h^{-d+1} \\
& +R_{h s}^{\prime}(h, l, b) .
\end{aligned}
$$

with

$$
\left|R_{h s}^{\prime}(h, l, b)\right| \leq C l^{d-2} h^{-d+2}\left(1+l^{2} h^{-2}|b|(1+|\ln | b||)\right) .
$$

Here the constants $C>0$ depend only on $d$ and $C_{\phi}$.

Remark The proposition shows, in particular, that $L_{d}^{(2)}(b)$ is non-increasing. Indeed, for given boundary coefficients $b \leq b^{\prime}$ the variational principle implies $\operatorname{Tr}(H(b))_{-} \geq$ $\operatorname{Tr}\left(H\left(b^{\prime}\right)\right)_{-}$for all $h>0$, and Proposition 3.1 thus yields $L_{d}^{(2)}(b) \geq L_{d}^{(2)}\left(b^{\prime}\right)$.

The first part of Proposition 3.1 is the key semi-classical estimate that we will later generalize to curved boundaries and variable $b$ 's. The problem with this bound, however, is the $|b|^{-1}$ in the error term which blows up for small values of $b$. For that reason we need to include the second part, which deals with small values of $b$. (In passing, we note that since $L_{d}^{(2)}(b)$ is continuously differentiable with $L_{d}^{(2)}(0)=\frac{1}{4} L_{d-1}^{(1)}$, as we will see in Lemma 3.5, the constant $\frac{1}{4} L_{d-1}^{(1)}$ in the second part of Proposition 3.1 can be replaced by $L_{d}^{(2)}(b)$ without changing the form of the error term.)

To deal with the transition region between $|b| \geq 1$ (where the first part of Proposition 3.1 applies) and $|b| \leq h / l$ (where the second part applies) we need the following rough estimate.

Lemma 3.2 Assume that $b \in \mathbb{R}$ is constant. Let $\phi \in C_{0}^{1}\left(\mathbb{R}^{d}\right)$ be supported in a ball of radius $l>0$ and let (2.4) be satisfied. Then for all $0<h \leq l$ we have

$$
\operatorname{Tr}\left(\phi H^{+}(b) \phi\right)_{-}=L_{d}^{(1)} \int_{\mathbb{R}_{+}^{d}} \phi^{2}(x) d x h^{-d}+R_{h s}^{\prime \prime}(h, l, b)
$$

with

$$
\left|R_{h s}^{\prime \prime}(h, l, b)\right| \leq C l^{d-1} h^{-d+1}\left(1+b_{-}^{d+1} \min \left\{b_{-} l h^{-1}, 1\right\}\right) .
$$

Here $C>0$ depends only on $d$ and $C_{\phi}$.

From this lemma we immediately deduce a simple bound that will be useful in the following sections.

Corollary 3.3 Assume that $b \in \mathbb{R}$ is constant. Let $\phi \in C_{0}^{1}\left(\mathbb{R}^{d}\right)$ be supported in a ball of radius $l>0$ and let (2.4) be satisfied. Then for all $0<h \leq l$ the bound 


$$
\operatorname{Tr}\left(\phi H^{+}(b) \phi\right)_{-} \leq C l^{d} h^{-d}\left(1+b_{-}^{d+1} h l^{-1}\right)
$$

holds with a constant $C$ depending only on $d$ and $C_{\phi}$.

The next remark will be used at several places without explicit mentioning in the proofs of Proposition 3.1 and Lemma 3.2.

Remark When bounding error terms in the following proofs we will sometimes encounter the term $\|\phi\|_{\infty}$, which is not mentioned in Proposition 3.1 and elsewhere. The reason is that it can be controlled in terms of $C_{\phi}$. Indeed, for $x$ in the support of $\phi$ we can choose $y$ at the boundary of the support with $|x-y| \leq l$ and use (2.4) to estimate

$$
|\phi(x)|=|\phi(x)-\phi(y)| \leq\|\nabla \phi\|_{\infty}|x-y| \leq C_{\phi} .
$$

Hence, $\|\phi\|_{\infty} \leq C_{\phi}$, as claimed.

\subsection{Analysis of a model operator on the half-line}

The bounds in Proposition 3.1 and Lemma 3.2 are based on the following results about the one dimensional operator $-\frac{d^{2}}{d t^{2}}$ on the half-line $\mathbb{R}_{+}$with boundary condition

$$
\partial_{t} v(0)=b v(0), b \in \mathbb{R} .
$$

For $t \geq 0$ and $b \in \mathbb{R}$ we define

$$
\psi_{b}(t)=\frac{1}{\sqrt{1+b^{2}}} \cos (t)+\frac{b}{\sqrt{1+b^{2}}} \sin (t)
$$

and, for $b<0$,

$$
\Psi_{b}(t)=\sqrt{-2 b} e^{b t}
$$

In order to treat positive and negative $b$ without distinction we set $\Psi_{b} \equiv 0$ for $b \geq 0$. Then we have

$$
\begin{aligned}
& -\partial_{t}^{2} \psi_{b}(t)=\psi_{b}(t), \\
& -\partial_{t}^{2} \Psi_{b}(t)=-b^{2} \Psi_{b}(t),
\end{aligned}
$$

and all functions satisfy boundary conditions (3.1). These functions form a complete system of (generalized) eigenfunctions: For functions $v \in L^{2}\left(\mathbb{R}_{+}\right)$we have

$$
v(t)=\int_{0}^{\infty}\left(\frac{2}{\pi} \int_{0}^{\infty} \psi_{b / p}(t p) \psi_{b / p}(s p) d p+\Psi_{b}(t) \Psi_{b}(s)\right) v(s) d s
$$


in the sense of $L^{2}$-convergence. This identity holds for continuous $v \in L^{1}\left(\mathbb{R}_{+}\right) \cap$ $L^{2}\left(\mathbb{R}_{+}\right)$and is extended first to $L^{1}\left(\mathbb{R}_{+}\right) \cap L^{2}\left(\mathbb{R}_{+}\right)$and then to $L^{2}\left(\mathbb{R}_{+}\right)$as in the case of the ordinary Fourier transform.

We need the following technical result.

Lemma 3.4 For $t \in \mathbb{R}_{+}$and $b \in \mathbb{R}$ we have

$$
\psi_{b}^{2}(t) \leq 1
$$

Moreover, the function

$$
I_{b}(t)=\int_{0}^{1}\left(1-p^{2}\right)^{(d+1) / 2}\left(\frac{p^{2}-b^{2}}{p^{2}+b^{2}} \cos (2 t p)+\frac{2 p b}{p^{2}+b^{2}} \sin (2 t p)\right) d p
$$

is uniformly bounded with respect to $t \geq 0$ and $b \in \mathbb{R}$. It satisfies

$$
\int_{0}^{\infty}\left|I_{b}(t)\right| d t \leq C \text { and } \int_{0}^{\infty} t\left|I_{b}(t)\right| d t \leq C \times \begin{cases}1 & \text { if } b=0 \\ \left(1+\frac{1}{|b|}\right) & \text { if } b \neq 0\end{cases}
$$

with $C>0$ depending only on the dimension.

Proof The first assertion follows directly from the definition of $\psi_{b}$ since

$$
\psi_{b}^{2}(t)=\frac{1}{2}+\frac{\left(1-b^{2}\right) \cos (2 t)+2 b \sin (2 t)}{2\left(1+b^{2}\right)}=\frac{1}{2}+\frac{(1-i b)^{2} e^{i 2 t}+(1+i b)^{2} e^{-i 2 t}}{4\left(1+b^{2}\right)} .
$$

It is clear from the definition that $I_{b}$ is uniformly bounded. To establish decay in $t$ we write

$$
I_{b}(t)=\frac{1}{2} \int_{\mathbb{R}}\left(1-p^{2}\right)_{+}^{(d+1) / 2} \frac{(p-i b)^{2}}{p^{2}+b^{2}} e^{i 2 t p} d p
$$

and set $G(p)=\left(1-p^{2}\right)_{+}^{(d+1) / 2}$ and $H_{b}(p)=(p-i b)^{2} /\left(p^{2}+b^{2}\right)$. Let $\check{G}$ and $\check{H}_{b}$ denote the inverse (distributional) Fourier transforms of $G$ and $H_{b}$.

It is well known that $\breve{G}(t)=c_{d} J_{d / 2+1}(|t|)|t|^{-d / 2-1}$, where $J_{d / 2+1}$ denotes the Bessel function of the first kind. The absolute value of this Bessel function behaves like $t^{d / 2+1}$ as $t \rightarrow 0+$ and is bounded by a constant times $t^{-1 / 2}$ as $t \rightarrow \infty$; see [1, (9.1.7) and (9.2.1)]. Hence, we have $|\breve{G}(t)| \leq C \min \left\{1,|t|^{-(d+3) / 2}\right\}$. Moreover, we compute that

$$
\check{H}_{b}(t)=(2 \pi)^{1 / 2} \delta(t)-2^{3 / 2} \pi^{1 / 2}|b| \chi_{\mathbb{R}_{-}}(b t) e^{-|b t|} .
$$


Thus we may rewrite $I_{b}(t)$ in terms of $\check{G}$ and $\check{H}_{b}$ and get

$$
\begin{aligned}
I_{b}(t) & =\frac{1}{2} \int_{\mathbb{R}} \check{G}(2 t-u) \check{H}_{b}(u) d u \\
& =\left(\frac{\pi}{2}\right)^{1 / 2} \check{G}(2 t)-(2 \pi)^{1 / 2}|b| \int_{\mathbb{R}} \check{G}(2 t-u) \chi_{\mathbb{R}_{-}}(b u) e^{-|b u|} d u \\
& =\left(\frac{\pi}{2}\right)^{1 / 2} \check{G}(2 t)-(2 \pi)^{1 / 2} \int_{0}^{\infty} \check{G}\left(2 t+\frac{u}{b}\right) e^{-u} d u .
\end{aligned}
$$

In the last change of variables we have assumed that $b \neq 0$. From the bound $|\check{G}(t)|=$ $|\hat{G}(-t)| \leq C \min \left\{1,|t|^{-(d+3) / 2}\right\}$ we easily derive that $\int_{0}^{\infty}|\breve{G}(2 t+u / b)| d t \leq C$. Moreover,

$$
\begin{aligned}
\int_{0}^{\infty} t|\check{G}(2 t+u / b)| d t & =\frac{1}{4} \int_{u / b}^{\infty}(t-u / b)|\check{G}(t)| d t \\
& \leq \frac{1}{4}\left(\int_{\mathbb{R}}|t||\check{G}(t)| d t+\frac{u}{|b|} \int_{\mathbb{R}}|\check{G}(t)| d t\right) \leq C\left(1+\frac{u}{|b|}\right) .
\end{aligned}
$$

This implies (3.5) for $b \neq 0$. The case $b=0$ is similar.

The next lemma establishes a connection between the function $I_{b}$ and the coefficient $L_{d}^{(2)}(b)$ defined in (1.11).

Lemma 3.5 For $L_{d}^{(2)}(b)$ we have the representations

$$
L_{d}^{(2)}(b)= \begin{cases}C_{d} \int_{0}^{\infty} I_{b}(t) d t & \text { for } b \geq 0 \\ C_{d}\left(\int_{0}^{\infty} I_{b}(t) d t+\pi\left(b^{2}+1\right)^{(d+1) / 2}\right) & \text { for } b<0\end{cases}
$$

The function $b \mapsto L_{d}^{(2)}(b)$ is continuously differentiable.

Proof Because of the first bound in (3.5) we may apply the dominated convergence theorem to write

$$
\begin{aligned}
\int_{0}^{\infty} I_{b}(t) d t= & \lim _{\epsilon \downarrow 0} \int_{0}^{1}\left(1-p^{2}\right)^{(d+1) / 2} \\
& \times \int_{0}^{\infty} e^{-\epsilon t^{2}}\left(\frac{p^{2}-b^{2}}{p^{2}+b^{2}} \cos (2 t p) d t+\frac{2 p b}{p^{2}+b^{2}} \sin (2 t p)\right) d t d p
\end{aligned}
$$




$$
\begin{aligned}
=\lim _{\epsilon \downarrow 0}\left(\frac{\sqrt{\pi}}{2} \int_{0}^{1 / \sqrt{\epsilon}}\left(1-\epsilon q^{2}\right)^{(d+1) / 2} \frac{\epsilon q^{2}-b^{2}}{\epsilon q^{2}+b^{2}} e^{-q^{2}} d q\right. \\
\left.+\int_{0}^{1}\left(1-p^{2}\right)^{(d+1) / 2} \frac{2 p b}{p^{2}+b^{2}} \frac{1}{\sqrt{\epsilon}} F\left(\frac{p}{\sqrt{\epsilon}}\right) d p\right),
\end{aligned}
$$

where $F(x)=e^{-x^{2}} \int_{0}^{x} e^{y^{2}} d y$. Using the fact that

$$
\lim _{\epsilon \downarrow 0} \frac{1}{\sqrt{\epsilon}} F\left(\frac{p}{\sqrt{\epsilon}}\right)=\frac{1}{2 p}
$$

we find

$$
\int_{0}^{\infty} I_{b}(t) d t=-\frac{\pi}{4}+\int_{0}^{1}\left(1-p^{2}\right)^{(d+1) / 2} \frac{b}{b^{2}+p^{2}} d p
$$

for $b \neq 0$ and $\int_{0}^{\infty} I_{b}(t) d t=\frac{\pi}{4}$ for $b=0$. By (1.11) this yields (3.7).

The fact that $b \mapsto L_{d}^{(2)}(b)$ is $C^{1}$ away from $b=0$ is elementary. To prove continuity and differentiability at $b=0$ we again use dominated convergence together with the fact that

$$
\lim _{b \rightarrow 0 \pm} \int_{0}^{1}\left(1-p^{2}\right)^{(d+1) / 2} \frac{b}{b^{2}+p^{2}} d p= \pm \frac{\pi}{2} .
$$

We omit the details.

\subsection{Proof of Propositions 3.1 and Lemma 3.2}

After these preliminaries we can turn to the proof of local asymptotics on the halfspace. We split the proof into three lemmas.

Lemma 3.6 Under the conditions of Proposition 3.1 we have

$$
\begin{aligned}
0 \leq & 2 C_{d} \int_{\mathbb{R}_{+}^{d}} \int_{0}^{1} \phi^{2}(x)\left(1-\xi_{d}^{2}\right)^{(d+1) / 2} \psi_{b / \xi_{d}}^{2}\left(x_{d} \xi_{d} / h\right) d \xi_{d} d x h^{-d} \\
& +\pi C_{d}\left(b^{2}+1\right)^{(d+1) / 2} \int_{\mathbb{R}_{+}^{d}} \phi^{2}(x) \Psi_{b / h}^{2}\left(x_{d}\right) d x h^{-d+1}-\operatorname{Tr}\left(\phi H^{+}(b) \phi\right)_{-} \\
\leq & C l^{d-2} h^{-d+2}\left(1+b_{-}^{d-1} \min \left\{b_{-}, h / l\right\}\right)
\end{aligned}
$$

where $C_{d}$ is given in (1.11). Here the constant $C>0$ depends only on $d$ and $C_{\phi}$. 
Proof First note that we may rescale $\phi$ and thus assume $l=1$ without changing the value of $b$. Since $b$ is fixed throughout the proof we write $H^{+}$instead of $H^{+}(b)$.

To prove the lower bound we apply the variational principle and obtain

$-\operatorname{Tr}\left(\phi H^{+} \phi\right)_{-}=\inf _{0 \leq \gamma \leq 1} \operatorname{Tr}\left(\gamma \phi H^{+} \phi\right) \geq \inf _{0 \leq \gamma \leq 1}\left(-\operatorname{Tr}\left(\gamma \phi\left(H^{+}\right)_{-} \phi\right)\right)=-\operatorname{Tr}\left(\phi\left(H^{+}\right)_{-} \phi\right)$.

Let $a^{+}(x, y)$ denote the integral kernel of $\left(H^{+}\right)_{-}$. From (3.2), (3.3), and (3.4) we see that

$$
\begin{aligned}
a^{+}(x, y)= & \frac{4}{(2 \pi h)^{d}} \int_{\mathbb{R}_{+}^{d}}\left(|\xi|^{2}-1\right)_{-} e^{i \xi^{\prime} \cdot\left(x^{\prime}-y^{\prime}\right) / h} \psi_{b / \xi_{d}}\left(x_{d} \xi_{d} / h\right) \psi_{b / \xi_{d}}\left(y_{d} \xi_{d} / h\right) d \xi \\
& +\frac{1}{(2 \pi h)^{d-1}} \int_{\mathbb{R}^{d-1}}\left(\left|\xi^{\prime}\right|^{2}-b^{2}-1\right)_{-} e^{i \xi^{\prime} \cdot\left(x^{\prime}-y^{\prime}\right) / h} \Psi_{b / h}\left(x_{d}\right) \Psi_{b / h}\left(y_{d}\right) d \xi^{\prime}
\end{aligned}
$$

and we get

$$
\begin{aligned}
\operatorname{Tr}\left(\phi H^{+} \phi\right)_{-} \leq & \frac{4}{(2 \pi h)^{d}} \int_{\mathbb{R}_{+}^{d}} \int_{\mathbb{R}_{+}^{d}} \phi^{2}(x)\left(|\xi|^{2}-1\right)_{-} \psi_{b / \xi_{d}}^{2}\left(x_{d} \xi_{d} / h\right) d \xi d x \\
& +\frac{1}{(2 \pi h)^{d-1}} \int_{\mathbb{R}_{+}^{d}} \int_{\mathbb{R}^{d-1}} \phi^{2}(x)\left(\left|\xi^{\prime}\right|^{2}-b^{2}-1\right)_{-} \Psi_{b / h}^{2}\left(x_{d}\right) d \xi^{\prime} d x
\end{aligned}
$$

Here we perform the $\xi^{\prime}$-integration and obtain the lower bound.

We proceed to prove the upper bound. To simplify notation write

$$
\begin{aligned}
f(x, \xi) & =e^{i x^{\prime} \cdot \xi^{\prime}} \psi_{b /\left(\xi_{d} h\right)}\left(x_{d} \xi_{d}\right) \\
F\left(x, \xi^{\prime}\right) & =e^{i x^{\prime} \cdot \xi^{\prime}} \Psi_{b / h}\left(x_{d}\right)
\end{aligned}
$$

We define the operator $\gamma=\left(H^{+}\right)_{-}^{0}$ with kernel $\gamma(x, y)=\gamma_{1}(x, y)+\gamma_{2}(x, y)$, where

$$
\begin{aligned}
& \gamma_{1}(x, y)=\frac{4}{(2 \pi h)^{d}} \int_{\left\{\xi \in \mathbb{R}_{+}^{d}:|\xi|<1\right\}} f(x, \xi / h) \overline{f(y, \xi / h)} d \xi \\
& \gamma_{2}(x, y)=\frac{1}{(2 \pi h)^{d-1}} \int_{\left\{\xi^{\prime} \in \mathbb{R}^{d-1}:\left|\xi^{\prime}\right|^{2}<b^{2}+1\right\}} F\left(x, \xi^{\prime} / h\right) \overline{F\left(y, \xi^{\prime} / h\right)} d \xi^{\prime} .
\end{aligned}
$$

Thus, $\gamma$ satisfies $0 \leq \gamma \leq 1$ and a variant of the variational principle, discussed in Appendix B, yields

$$
-\operatorname{Tr}\left(\phi H^{+} \phi\right)_{-} \leq \operatorname{Tr}\left(\phi \gamma \phi H^{+}\right)=\operatorname{Tr}\left(\phi \gamma_{1} \phi H^{+}\right)+\operatorname{Tr}\left(\phi \gamma_{2} \phi H^{+}\right)
$$


We note that the range of $\phi \gamma \phi, \phi \gamma_{1} \phi$, and $\phi \gamma_{2} \phi$ does not belong to the domain of $H^{+}$. However, the functions $\phi f$ and $\phi F$ belong to the form domain $H^{1}\left(\mathbb{R}_{+}^{d}\right)$ of $H^{+}$. Therefore (3.8) is valid if we interpret $\operatorname{Tr}\left(\phi \gamma \phi H^{+}\right)$in the sense described in Appendix B, namely

$$
\operatorname{Tr}\left(\phi \gamma_{1} \phi H^{+}\right)=\frac{4}{(2 \pi h)^{d}} \int_{\left\{\xi \in \mathbb{R}_{+}^{d}:|\xi|<1\right\}} q_{b}^{+}[\phi f] d \xi
$$

where

$$
q_{b}^{+}[\phi f]=h^{2}\|\nabla(\phi f)\|_{L^{2}\left(\mathbb{R}_{+}^{d}\right)}^{2}+h b\|\phi(\cdot, 0)\|_{L^{2}\left(\mathbb{R}^{d-1}\right)}^{2} \psi_{b / \xi_{d}}^{2}(0)-\|\phi f\|_{L^{2}\left(\mathbb{R}_{+}^{d}\right)}^{2},
$$

and similar for $\operatorname{Tr}\left(\phi \gamma_{2} \phi H^{+}\right)$. In the first summand we integrate by parts and use (3.1) and (3.2) to get

$$
\begin{aligned}
\|\nabla(\phi f)\|_{L^{2}\left(\mathbb{R}_{+}^{d}\right)}^{2}= & \int_{\mathbb{R}_{+}^{d}}\left(\frac{|\xi|^{2}}{h^{2}} \phi^{2}+|\nabla \phi|^{2}\right) \psi_{b / \xi_{d}}^{2}\left(x_{d} \xi_{d} / h\right) d x \\
& -\frac{b}{h}\|\phi(\cdot, 0)\|_{L^{2}\left(\mathbb{R}^{d-1}\right)}^{2} \psi_{b / \xi_{d}}^{2}(0) .
\end{aligned}
$$

We insert this into (3.9) and due to (2.4) and Lemma 3.4 we can estimate

$$
\operatorname{Tr}\left(\phi \gamma_{1} \phi H^{+}\right) \leq-\frac{4}{(2 \pi h)^{d}} \int_{\mathbb{R}_{+}^{d}} \int_{\mathbb{R}_{+}^{d}} \phi^{2}(x)\left(|\xi|^{2}-1\right)_{-} \psi_{b / \xi_{d}}^{2}\left(x_{d} \xi_{d} / h\right) d x d \xi+C h^{-d+2}
$$

Note that the second summand in (3.8) is zero for $b \geq 0$. For $b<0$ we use (3.1) and (3.3) to show that

$$
\begin{aligned}
\operatorname{Tr}\left(\phi \gamma_{2} \phi H^{+}\right)= & \frac{1}{(2 \pi h)^{(d-1)}} \int_{\left\{\left|\xi^{\prime}\right|^{2}<b^{2}+1\right\}} q_{b}^{+}[\phi F] d \xi^{\prime} \\
= & \frac{1}{(2 \pi h)^{(d-1)}} \int_{\mathbb{R}^{d-1}} \int_{\mathbb{R}_{+}^{d}} \phi^{2}(x)\left(1+b^{2}-\left|\xi^{\prime}\right|^{2}\right)_{+} \Psi_{b / h}^{2}\left(x_{d}\right) d x d \xi^{\prime} \\
& +\frac{h^{2}}{(2 \pi h)^{(d-1)}} \int_{\left\{\left|\xi^{\prime}\right|^{2}<b^{2}+1\right\}} \int_{\mathbb{R}_{+}^{d}}|\nabla \phi(x)|^{2} \Psi_{b / h}^{2}\left(x_{d}\right) d x d \xi^{\prime}
\end{aligned}
$$


To estimate the last summand we use $\left\|\Psi_{b / h}\right\|_{\infty}^{2} \leq-2 b h^{-1},\left\|\Psi_{b / h}\right\|_{2}^{2}=1$, and (2.4) to obtain

$$
\int_{\mathbb{R}_{+}^{d}}|\nabla \phi(x)|^{2} \Psi_{b / h}^{2}\left(x_{d}\right) d x \leq C \min \{-b / h, 1\} .
$$

Performing the $\xi^{\prime}$-integration as before yields

$$
\begin{aligned}
\operatorname{Tr}\left(\phi \gamma_{2} \phi H^{+}\right) \leq & -\pi C_{d} h^{-d+1}\left(b^{2}+1\right)^{(d+1) / 2} \int_{\mathbb{R}_{+}^{d}} \phi^{2}(x) \Psi_{b / h}^{2}\left(x_{d}\right) d x \\
& +C h^{-d+2}\left(1+b_{-}^{d-1} \min \left\{h, b_{-}\right\}\right) .
\end{aligned}
$$

Here we also used the fact that $1+\left(1+b^{2}\right)^{(d-1) / 2} \min \left\{b_{-}, h\right\} \leq C\left(1+b_{-}^{d-1}\right.$ $\left.\min \left\{b_{-}, h\right\}\right)$. Hence, the upper bound follows from (3.8), (3.10), and (3.11).

Lemma 3.7 Under the conditions of Proposition 3.1 we have

$$
\begin{aligned}
& 2 C_{d} \int_{\mathbb{R}_{+}^{d}} \int_{0}^{1} \phi^{2}(x)\left(1-\xi_{d}^{2}\right)^{(d+1) / 2} \psi_{b / \xi_{d}}^{2}\left(x_{d} \xi_{d} / h\right) d \xi_{d} d x \\
& =L_{d}^{(1)} \int_{\mathbb{R}_{+}^{d}} \phi^{2}(x) d x+C_{d} \int_{0}^{\infty} I_{b}(t) d t \int_{\mathbb{R}^{d-1}} \phi^{2}\left(x^{\prime}, 0\right) d x^{\prime} h+r_{1}(h, b)
\end{aligned}
$$

with $\left|r_{1}(h, b)\right| \leq C(1+1 /|b|) l^{d-2} h^{2}$ for $b \neq 0$ and $\left|r_{1}(h, 0)\right| \leq C l^{d-2} h^{2}$. For $b<0$ we also have

$$
\int_{\mathbb{R}_{+}^{d}} \phi^{2}(x) \Psi_{b / h}^{2}\left(x_{d}\right) d x=\int_{\mathbb{R}^{d-1}} \phi^{2}\left(x^{\prime}, 0\right) d x^{\prime}+r_{2}(h, b)
$$

with $\left|r_{2}(h, b)\right| \leq C l^{d-2} h b_{-}^{-1}$. Here the constants $C>0$ depend only on $d$ and $C_{\phi}$.

Proof Recall that

$$
L_{d}^{(1)}=\frac{1}{(2 \pi)^{d}} \int_{\mathbb{R}^{d}}\left(|\xi|^{2}-1\right)_{-} d \xi=C_{d} \int_{0}^{1}\left(1-\xi_{d}\right)^{(d+1) / 2} d \xi_{d}
$$


Hence,

$$
\begin{aligned}
& 2 C_{d} \int_{\mathbb{R}_{+}^{d}} \int_{0}^{1} \phi^{2}(x)\left(1-\xi_{d}^{2}\right)^{(d+1) / 2} \psi_{b / \xi_{d}}^{2}\left(x_{d} \xi_{d} / h\right) d \xi_{d} d x \\
& =L_{d}^{(1)} \int_{\mathbb{R}_{+}^{d}} \phi^{2}(x) d x+C_{d} \iint_{\mathbb{R}_{+}^{d}} \int_{0}^{1} \phi^{2}(x)\left(1-\xi_{d}\right)^{(d+1) / 2}\left(2 \psi_{b / \xi_{d}}^{2}\left(x_{d} \xi_{d} / h\right)-1\right) d \xi_{d} d x .
\end{aligned}
$$

We insert (3.6) and perform the $\xi^{\prime}$ integration and see that the right-hand side equals

$$
L_{d}^{(1)} \int_{\mathbb{R}_{+}^{d}} \phi^{2}(x) d x+C_{d} \int_{\mathbb{R}_{+}^{d}} \phi^{2}(x) I_{b}\left(\frac{x_{d}}{h}\right) d x
$$

with $I_{b}$ introduced in Lemma 3.4. To analyze the second term we insert

$$
\phi^{2}(x)=\phi^{2}\left(x^{\prime}, x_{d}\right)=\phi^{2}\left(x^{\prime}, 0\right)+\int_{0}^{x_{d}} \partial_{s} \phi^{2}\left(x^{\prime}, s\right) d s
$$

and substitute $x_{d}=t h$. We obtain

$$
\begin{aligned}
& 2 C_{d} \int_{\mathbb{R}_{+}^{d}} \int_{0}^{1} \phi^{2}(x)\left(1-\xi_{d}^{2}\right)^{(d+1) / 2} \psi_{b / \xi_{d}}^{2}\left(x_{d} \xi_{d} / h\right) d \xi_{d} d x \\
& =L_{d}^{(1)} \int_{\mathbb{R}_{+}^{d}} \phi^{2}(x) d x+C_{d} \int_{0}^{\infty} I_{b}(t) d t \int_{\mathbb{R}^{d-1}} \phi^{2}\left(x^{\prime}, 0\right) d x^{\prime} h \\
& +C \int_{\mathbb{R}^{d-1}} \int_{0}^{\infty} \int_{0}^{t h} \partial_{s} \phi^{2}\left(x^{\prime}, s\right) d s I_{b}(t) d t d x^{\prime} h .
\end{aligned}
$$

Using (2.4) and the remark at the end of Sect. 3.1 we bound

$$
\left|\int_{\mathbb{R}^{d-1}} \int_{0}^{t h} \partial_{s} \phi^{2}\left(x^{\prime}, s\right) d s d x^{\prime}\right| \leq C l^{d-2} h t .
$$

The first assertion of the lemma now follows from (3.5).

The second assertion follows similarly by inserting (3.14) and by definition of $\Psi_{b}$. 
Note that the error terms in Lemma 3.7 diverge as $b \rightarrow 0$. Hence, we also need the following estimates that yield better results for $|b| \leq C h / l$.

Lemma 3.8 Under the conditions of Proposition 3.1 we have

$$
\begin{aligned}
& 2 C_{d} \int_{\mathbb{R}_{+}^{d}} \int_{0}^{1} \phi^{2}(x)\left(1-\xi_{d}^{2}\right)^{(d+1) / 2} \psi_{b / \xi_{d}}^{2}\left(x_{d} \xi_{d} / h\right) d \xi_{d} d x \\
& =L_{d}^{(1)} \int_{\mathbb{R}_{+}^{d}} \phi^{2}(x) d x+\frac{1}{4} L_{d-1}^{(1)} \int_{\mathbb{R}^{d-1}} \phi^{2}\left(x^{\prime}, 0\right) d x^{\prime} h+\tilde{r}_{1}(h, b)
\end{aligned}
$$

with $\left|\tilde{r}_{1}(h, b)\right| \leq C l^{d-2} h^{2}\left(1+l^{2} h^{-2}|b|(1+|\ln | b||)\right)$. For $b<0$ we also have

$$
0 \leq \int_{\mathbb{R}_{+}^{d}} \phi^{2}(x) \Psi_{b / h}^{2}\left(x_{d}\right) d x \leq C l^{d} h^{-1} \min \left\{b_{-}, h l^{-1}\right\}
$$

Here the constants $C>0$ depend only on $d$ and $C_{\phi}$.

Proof This proof is a variation of the previous one. Again, we write

$$
\begin{aligned}
& 2 C_{d} \int_{\mathbb{R}_{+}^{d}} \int_{0}^{1} \phi^{2}(x)\left(1-\xi_{d}^{2}\right)^{(d+1) / 2} \psi_{b / \xi_{d}}^{2}\left(x_{d} \xi_{d} / h\right) d \xi_{d} d x=L_{d}^{(1)} \int_{\mathbb{R}_{+}^{d}} \phi^{2}(x) d x \\
& +C_{d} \int_{\mathbb{R}_{+}^{d}} \phi^{2}(x) I_{b}\left(\frac{x_{d}}{h}\right) d x
\end{aligned}
$$

We add and subtract $I_{0}$ to and from $I_{b}$. According to the previous lemma and Lemma 3.5 we have

$$
\left|C_{d} \int_{\mathbb{R}_{+}^{d}} \phi^{2}(x) I_{0}\left(\frac{x_{d}}{h}\right) d x-\frac{1}{4} L_{d-1}^{(1)} \int_{\mathbb{R}^{d-1}} \phi^{2}\left(x^{\prime}, 0\right) d x^{\prime} h\right| \leq C l^{d-2} h^{2}
$$

Thus, it remains to control

$$
C_{d} \int_{\mathbb{R}_{+}^{d}} \phi^{2}(x)\left(I_{b}\left(\frac{x_{d}}{h}\right)-I_{0}\left(\frac{x_{d}}{h}\right)\right) d x
$$


Recalling the definitions of $I_{b}$ and $I_{0}$ we see that the absolute value of this term is bounded by

$$
C \int_{\mathbb{R}_{+}^{d}} \phi^{2}(x) d x \int_{0}^{1}\left(1-p^{2}\right)^{(d+1) / 2} \frac{b^{2}+|b| p}{p^{2}+b^{2}} d p \leq C l^{d}|b|(1+|\ln | b||) .
$$

This finishes the proof of the first assertion of the lemma. The second assertion follows similarly as at the end of the proof of Lemma 3.6

Proof of Proposition 3.1 Combining Lemma 3.6 with (3.12), (3.13), and (3.7) we obtain the first claim of Proposition 3.1 with a remainder

$$
\begin{aligned}
\left|R_{h s}(h, l, b)\right| & \leq C l^{d-2} h^{-d+2}\left(1+|b|^{-1}+\left(b^{2}+1\right)^{(d+1) / 2} b_{-}^{-1}+b_{-}^{d-1} \min \left\{b_{-}, h l^{-1}\right\}\right) \\
& \leq C l^{d-2} h^{-d+2}|b|^{-1}\left(1+|b|+b_{-}^{d+1}\right) .
\end{aligned}
$$

To obtain the second claim we combine Lemma 3.6 with Lemma 3.8. In this case the remainder is bounded by a constant times

$l^{d-2} h^{-d+2}\left(1+l^{2} h^{-2}|b|(1+|\ln | b||)+\left(\left(b^{2}+1\right)^{(d+1) / 2} l^{2} h^{-2}+b_{-}^{d-1}\right) \min \left\{b_{-}, h / l\right\}\right)$.

For $|b| \leq h / l \leq 1$ this simplifies to

$$
\left|R_{h s}^{\prime}(h, l, b)\right| \leq C l^{d-2} h^{-d+2}\left(1+l^{2} h^{-2}|b|(1+|\ln | b \mid)\right) .
$$

This finishes the proof of the proposition.

Proof of Lemma 3.2 Combining Lemma 3.6 with (3.15) we obtain the claim with a remainder bounded by

$$
\begin{aligned}
\left|R_{h s}^{\prime \prime}(h, l, b)\right|= & C_{d} \int_{\mathbb{R}_{+}^{d}} \phi^{2}(x)\left|I_{b}\left(\frac{x_{d}}{h}\right)\right| d x h^{-d} \\
& +\pi C_{d}\left(b^{2}+1\right)^{(d+1) / 2} \int_{\mathbb{R}_{+}^{d}} \phi^{2}(x) \Psi_{b / h}^{2}\left(x_{d}\right) d x h^{-d+1} \\
& +C l^{d-2} h^{-d+2}\left(1+b_{-}^{d-1} \min \left\{b_{-}, h l^{-1}\right\}\right) .
\end{aligned}
$$

In the first term on the right side we substitute $x_{d}=t h$ and use the first inequality in (3.5) to bound

$$
\int_{0}^{\infty} \int_{\mathbb{R}^{d-1}} \phi^{2}\left(x^{\prime}, t h\right) d x^{\prime}\left|I_{b}(t)\right| d t \leq C l^{d-1}
$$


By Lemma 3.8 we also have

$$
0 \leq \int_{\mathbb{R}_{+}^{d}} \phi^{2}(x) \Psi_{b / h}^{2}\left(x_{d}\right) d x \leq C l^{d} h^{-1} \min \left\{b_{-}, h l^{-1}\right\}
$$

and the proof is complete.

\section{Local asymptotics close to the boundary}

Here we show how Proposition 2.3 and Lemma 2.4 follow from the results in Sect. 3. We straighten the boundary locally and estimate the operator $H(b)$ given on $\Omega$ in terms of $\mathrm{H}^{+}(b)$ given on the half-space $\mathbb{R}_{+}^{d}$.

In this section we work under the conditions of Proposition 2.3: Let $\phi \in C_{0}^{1}\left(\mathbb{R}^{d}\right)$ be supported in a ball of radius $l>0$ and let inequalities (1.10) and (2.4) be satisfied. Then let $B$ denote the open ball of radius $l>0$, containing the support of $\phi$. Choose $x_{0} \in B \cap \partial \Omega$ and let $v_{x_{0}}$ be the inner normal unit vector at $x_{0}$. We choose a Cartesian coordinate system such that $x_{0}=0$ and $v_{x_{0}}=(0, \ldots, 0,1)$.

We now introduce new local coordinates near the boundary. Let $D$ denote the projection of $B$ on the hyperplane given by $x_{d}=0$. Since the boundary of $\Omega$ is compact and in $C^{1}$, there is a constant $C_{\Omega}>0$, independent of $x_{0} \in \partial \Omega$, such that for $0<l \leq C_{\Omega}^{-1}$ we can find a real function $f \in C^{1}$, given on $D \subset \mathbb{R}^{d-1}$, satisfying

$$
\partial \Omega \cap B=\left\{\left(x^{\prime}, x_{d}\right): x^{\prime} \in D, x_{d}=f\left(x^{\prime}\right)\right\} \cap B .
$$

The fact that $\partial \Omega \in C^{1}$ means that the functions $\nabla f$ corresponding to different points $x_{0}$ and different values of $l$ share a common modulus of continuity which we denote by $\omega$, that is,

$$
\left|\nabla f\left(x^{\prime}\right)-\nabla f\left(y^{\prime}\right)\right| \leq \omega\left(\left|x^{\prime}-y^{\prime}\right|\right)
$$

for all $x^{\prime}, y^{\prime} \in D$. We assume that $\omega$ is non-decreasing and we emphasize that $\omega(\delta) \downarrow 0$ as $\delta \downarrow 0$.

The choice of coordinates implies $f(0)=0$ and $\nabla f(0)=0$. Hence, we can estimate

$$
\sup _{x^{\prime} \in D}\left|\nabla f\left(x^{\prime}\right)\right| \leq \sup _{x^{\prime} \in D} \omega\left(\left|x^{\prime}\right|\right) \leq \omega(l)
$$

We introduce new local coordinates given via a diffeomorphism $\varphi: D \times \mathbb{R} \rightarrow \mathbb{R}^{d}$. We set $y_{j}=\varphi_{j}(x)=x_{j}$ for $j=1, \ldots, d-1$ and $y_{d}=\varphi_{d}(x)=x_{d}-f\left(x^{\prime}\right)$. Note that the determinant of the Jacobian matrix of $\varphi$ equals 1 and that the inverse of $\varphi$ is defined on $\operatorname{ran} \varphi=D \times \mathbb{R}$. In particular, we get

$$
\varphi(\partial \Omega \cap B) \subset \partial \mathbb{R}_{+}^{d}=\left\{y \in \mathbb{R}^{d}: y_{d}=0\right\} .
$$


Fix $v \in H^{1}(\Omega)$ with $v \equiv 0$ on $\mathbb{R}^{d} \backslash \bar{B}$. For $y \in \operatorname{ran} \varphi$ put $\tilde{v}(y)=v \circ \varphi^{-1}(y)$ and extend $\tilde{v}$ by zero to $\mathbb{R}^{d}$. An explicit calculation shows that the effect of this change of coordinates on the gradient is small:

Lemma 4.1 For $v$ and $\tilde{v}$ defined as above we have $\tilde{v} \in H^{1}\left(\mathbb{R}_{+}^{d}\right)$ and

$$
\left.\left|\int_{\Omega}\right| \nabla v(x)\right|^{2} d x-\left.\int_{\mathbb{R}_{+}^{d}}|\nabla \tilde{v}(y)|^{2} d y\left|\leq C \omega(l) \int_{\mathbb{R}_{+}^{d}}\right| \nabla \tilde{v}(y)\right|^{2} d y .
$$

Based on this estimate we now prove a result from which Proposition 2.3 follows. For $\phi \in C_{0}^{\infty}\left(\mathbb{R}^{d}\right)$ supported in $B$ define $\tilde{\phi}=\phi \circ \varphi^{-1}$ on $\operatorname{ran} \varphi=D \times \mathbb{R}$ and extend it by zero to $\mathbb{R}^{d}$. It follows that $\tilde{\phi} \in C_{0}^{1}\left(\mathbb{R}^{d}\right)$ and $\|\nabla \tilde{\phi}\|_{\infty} \leq C l^{-1}$ hold, with $C$ depending only on $C_{\phi}$ and $\omega$. We set $b^{-}=\inf _{x \in \partial \Omega \cap B} b(x)$ and $b^{+}=\sup _{x \in \partial \Omega \cap B} b(x)$ and note that $\left(b^{+}\right)_{-} \leq\left(b^{-}\right)_{-} \leq b^{s}$, where $b^{s}$ was introduced in (2.6). We also recall the notation $H^{+}\left(b^{ \pm}\right)$introduced in Sect. 3 .

Lemma 4.2 Under the conditions of Proposition 2.3 there is a constant $C_{\Omega}>0$ depending only on $\Omega$ such that for $0<l \leq C_{\Omega}^{-1}$ and $0<h \leq l$ we have

$$
\begin{aligned}
& \operatorname{Tr}\left(\tilde{\phi} H^{+}\left(b^{+}\right) \tilde{\phi}\right)_{-}-C l^{d} h^{-d} \omega(l)\left(1+\left(b^{+}\right)_{-}^{d+1} h l^{-1}\right) \\
& \quad \leq \operatorname{Tr}(\phi H(b) \phi)_{-} \\
& \quad \leq \operatorname{Tr}\left(\tilde{\phi} H^{+}\left(b^{-}\right) \tilde{\phi}\right)_{-}+C l^{d} h^{-d} \omega(l)\left(1+\left(b^{-}\right)_{-}^{d+1} h l^{-1}\right) .
\end{aligned}
$$

Moreover,

$$
\begin{gathered}
\int_{\Omega} \phi^{2}(x) d x=\int_{\mathbb{R}_{+}^{d}} \tilde{\phi}^{2}(y) d y \\
\left|\int_{\partial \Omega} \phi^{2}(x) d \sigma(x)-\int_{\mathbb{R}^{d-1}} \tilde{\phi}^{2}\left(y^{\prime}, 0\right) d y^{\prime}\right| \leq C l^{d-1} \omega(l)^{2},
\end{gathered}
$$

and

$$
\begin{aligned}
& \left|\int_{\partial \Omega} L_{d}^{(2)}(b(x)) \phi^{2}(x) d \sigma(x)-L_{d}^{(2)}\left(b^{ \pm}\right) \int_{\mathbb{R}^{d-1}} \tilde{\phi}^{2}\left(y^{\prime}, 0\right) d y^{\prime}\right| \\
& \leq C l^{d-1}\left(\left(1+\left(b^{ \pm}\right)_{-}^{d+1}\right) \omega(l)^{2}+\left(1+\left(b^{ \pm}\right)_{-}^{d}\right) \beta(l)\right) .
\end{aligned}
$$


Proof The definition of $\tilde{\phi}$ and the fact that $\operatorname{det} J \varphi=1$ immediately give (4.4). In view of (4.1) we can estimate

$$
\int_{\partial \Omega} \phi^{2}(x) d \sigma(x)=\int_{\mathbb{R}^{d-1}} \tilde{\phi}^{2}\left(y^{\prime}, 0\right) \sqrt{1+|\nabla f|^{2}} d y^{\prime} \leq \int_{\mathbb{R}^{d-1}} \tilde{\phi}^{2}\left(y^{\prime}, 0\right) d y^{\prime}+C l^{d-1} \omega(l)^{2} .
$$

This proves (4.5). Using the fact that $\left|L_{d}^{(2)}\left(b^{ \pm}\right)\right| \leq C\left(1+\left(b^{ \pm}\right)_{-}^{d+1}\right)$ we find

$$
\begin{aligned}
& \left|\int_{\partial \Omega} L_{d}^{(2)}(b(x)) \phi^{2}(x) d \sigma(x)-L_{d}^{(2)}\left(b^{ \pm}\right) \int_{\mathbb{R}^{d-1}} \tilde{\phi}^{2}\left(y^{\prime}, 0\right) d y^{\prime}\right| \\
& \quad \leq \int_{\partial \Omega}\left|L_{d}^{(2)}(b(x))-L_{d}^{(2)}\left(b^{ \pm}\right)\right| \phi^{2}(x) d \sigma(x)+C l^{d-1} \omega(l)^{2}\left(1+\left(b^{ \pm}\right)_{-}^{d+1}\right) .
\end{aligned}
$$

The continuity of $b$, see (1.10), and the fact that $\left|\frac{d}{d b} L_{d}^{(2)}(b)\right| \leq C\left(1+b_{-}^{d}\right)$ imply

$$
\left|L_{d}^{(2)}\left(b^{ \pm}\right)-L_{d}^{(2)}(b(x))\right| \leq C \beta(l)\left(1+\left(b^{ \pm}\right)_{-}^{d}\right) .
$$

Inserting this into the estimate above gives (4.6).

To prove (4.3) we first note that the variational principle implies

$$
\operatorname{Tr}\left(\phi H\left(b^{+}\right) \phi\right)_{-} \leq \operatorname{Tr}(\phi H(b) \phi)_{-} \leq \operatorname{Tr}\left(\phi H\left(b^{-}\right) \phi\right)_{-} .
$$

Thus it remains to show that

$$
\left|\operatorname{Tr}\left(\phi H\left(b^{ \pm}\right) \phi\right)_{-}-\operatorname{Tr}\left(\tilde{\phi} H^{+}\left(b^{ \pm}\right) \tilde{\phi}\right)_{-}\right| \leq C l^{d} h^{-d} \omega(l)\left(1+\left(b^{ \pm}\right)_{-}^{d+1} h l^{-1}\right) .
$$

To this end choose $v$ and $\tilde{v}$ as in Lemma 4.1. First we estimate

$$
\int_{\partial \Omega}|v(x)|^{2} d \sigma(x)=\int_{\mathbb{R}^{d-1}}\left|\tilde{v}\left(y^{\prime}, 0\right)\right|^{2} \sqrt{1+|\nabla f|^{2}} d y^{\prime} \geq \int_{\mathbb{R}^{d-1}}\left|\tilde{v}\left(y^{\prime}, 0\right)\right|^{2} d y^{\prime} .
$$

and using (4.1)

$$
\int_{\partial \Omega}|v(x)|^{2} d \sigma(x) \leq\left(1+C \omega(l)^{2}\right) \int_{\mathbb{R}^{d-1}}\left|\tilde{v}\left(y^{\prime}, 0\right)\right|^{2} d y^{\prime} .
$$

By increasing, if necessary, the constant $C_{\Omega}$ from the beginning of this section we may now assume that $l>0$ is small enough such that $2 C \omega(l) \leq 1 / 2$ holds. Then Lemma 4.1, (4.4), and (4.8) imply, for $b^{ \pm} \geq 0$, 


$$
\begin{aligned}
q_{b^{ \pm}}[v] \geq & (1-C \omega(l)) h^{2} \int_{\mathbb{R}_{+}^{d}}|\nabla \tilde{v}(y)|^{2} d y+h b^{ \pm} \int_{\mathbb{R}^{d-1}}\left|\tilde{v}\left(y^{\prime}, 0\right)\right|^{2} d y^{\prime}-\int_{\mathbb{R}_{+}^{d}}|\tilde{v}(y)|^{2} d y \\
= & (1-2 C \omega(l)) q_{b^{ \pm}}^{+}[\tilde{v}] \\
& +2 C \omega(l)\left(\frac{h^{2}}{2} \int_{\mathbb{R}_{+}^{d}}|\nabla \tilde{v}(y)|^{2} d y+h b^{ \pm} \int_{\mathbb{R}^{d-1}}\left|\tilde{v}\left(y^{\prime}, 0\right)\right|^{2} d y^{\prime}-\int_{\mathbb{R}_{+}^{d}}|\tilde{v}(y)|^{2} d y\right) \\
= & (1-2 C \omega(l)) q_{b^{ \pm}}^{+}[\tilde{v}]+2 C \omega(l) \tilde{q}_{0}^{+}[\tilde{v}],
\end{aligned}
$$

where $\tilde{q}^{+}$is the same form as $q^{+}$but with $h$ replaced by $h / \sqrt{2}$. For $b^{ \pm}<0$ we get, using (4.9),

$$
\begin{aligned}
q_{b^{ \pm}}[v] \geq & (1-C \omega(l)) h^{2} \int_{\mathbb{R}_{+}^{d}}|\nabla \tilde{v}(y)|^{2} d y \\
& +\left(1+C \omega(l)^{2}\right) h b^{ \pm} \int_{\mathbb{R}^{d-1}}\left|\tilde{v}\left(y^{\prime}, 0\right)\right|^{2} d y^{\prime}-\int_{\mathbb{R}_{+}^{d}}|\tilde{v}(y)|^{2} d y \\
\geq & (1-2 C \omega(l)) q_{b^{ \pm}}^{+}[\tilde{v}]+2 C \omega(l) \tilde{q}_{C b^{ \pm}}^{+}[\tilde{v}] .
\end{aligned}
$$

To deduce estimates for $\operatorname{Tr}\left(\phi H\left(b^{ \pm}\right) \phi\right)_{-}$we recall the variational principle

$$
-\operatorname{Tr}\left(\phi H\left(b^{ \pm}\right) \phi\right)_{-}=\inf _{0 \leq \gamma \leq 1} \operatorname{Tr}\left(\phi \gamma \phi H\left(b^{ \pm}\right)\right)
$$

where we can assume that the infimum is taken over trial density matrices $\gamma$ supported in $\bar{B} \times \bar{B}$. Fix such a $\gamma$. For $y$ and $z$ from $D \times \mathbb{R}$ set

$$
\tilde{\gamma}(y, z)=\gamma\left(\varphi^{-1}(y), \varphi^{-1}(z)\right)
$$

so that $0 \leq \tilde{\gamma} \leq 1$ holds. Moreover, the range of $\tilde{\gamma}$ belongs to the form domain of $\tilde{\phi} H^{+}\left(b^{ \pm}\right) \tilde{\phi}$.

First, we assume $b^{ \pm}<0$. According to (4.11) it follows that

$$
\begin{aligned}
\operatorname{Tr}\left(\phi \gamma \phi H\left(b^{ \pm}\right)\right) & \geq \operatorname{Tr}\left(\tilde{\phi} \tilde{\gamma} \tilde{\phi}\left((1-2 C \omega(l)) H^{+}\left(b^{ \pm}\right)+2 C \omega(l) \tilde{H}^{+}\left(C b^{ \pm}\right)\right)\right) \\
& \geq-(1-2 C \omega(l)) \operatorname{Tr}\left(\tilde{\phi} H^{+}\left(b^{ \pm}\right) \tilde{\phi}\right)_{-}-2 C \omega(l) \operatorname{Tr}\left(\tilde{\phi} \tilde{H}^{+}\left(C b^{ \pm}\right) \tilde{\phi}\right)_{-},
\end{aligned}
$$

where the operator $\tilde{H}^{+}$is generated by the form $\tilde{q}^{+}$. This implies

$$
\operatorname{Tr}\left(\phi H\left(b^{ \pm}\right) \phi\right)_{-} \leq \operatorname{Tr}\left(\tilde{\phi} H^{+}\left(b^{ \pm}\right) \tilde{\phi}\right)_{-}+2 C \omega(l) \operatorname{Tr}\left(\tilde{\phi} \tilde{H}^{+}\left(C b^{ \pm}\right) \tilde{\phi}\right)_{-}
$$


and Corollary 3.3 yields

$$
\operatorname{Tr}\left(\phi H\left(b^{ \pm}\right) \phi\right)_{-} \leq \operatorname{Tr}\left(\tilde{\phi} H^{+}\left(b^{ \pm}\right) \tilde{\phi}\right)_{-}+C l^{d} h^{-d} \omega(l)\left(1+\left(b^{ \pm}\right)^{d+1} h / l\right)
$$

for $b^{ \pm}<0$.

In the same way we can treat non-negative $b^{ \pm}$using (4.10) and we obtain the lower bound in (4.7). Finally, by interchanging the roles of $H\left(b^{ \pm}\right)$and $H^{+}\left(b^{ \pm}\right)$, we get an analogous upper bound and the proof of Lemma 4.2 is complete.

Proof of Proposition 2.3 and Lemma 2.4 The assertions follow from Lemma 4.2 together with Proposition 3.1.

If we combine the estimates of Proposition 2.2, Corollary 3.3, and Lemma 4.2 we obtain the following simple bound that is useful to estimate error terms.

Corollary 4.3 There is a constant $C_{\Omega}>0$ with the following property. Let $\phi \in C_{0}^{\infty}$ be supported in a ball of radius $l>0$ and let (2.4) be satisfied. Assume that $b$ is $a$ real constant independent of $x$.

Then for $0<l \leq C_{\Omega}^{-1}$ and $0<h \leq l$ the estimate

$$
\operatorname{Tr}(\phi H(b) \phi)_{-} \leq C l^{d} h^{-d}\left(1+b_{-}^{d+1} h l^{-1}\right)
$$

holds with a constant $C>0$ depending only on $d, C_{\phi}$ and $\omega$.

\section{Localization}

In this section we construct the family of localization functions $\left(\phi_{u}\right)_{u \in \mathbb{R}^{d}}$ and prove Proposition 2.1. The key idea is to choose the localization depending on the distance to the complement of $\Omega$, see [6, Theorem 17.1.3] and [13] for a continuous version of this method.

Fix a real-valued function $\phi \in C_{0}^{\infty}\left(\mathbb{R}^{d}\right)$ with support in $\{|x|<1\}$ and $\|\phi\|_{2}=1$. For $u, x \in \mathbb{R}^{d}$ let $J(x, u)$ be the Jacobian of the map $u \mapsto(x-u) / l(u)$. We define

$$
\phi_{u}(x)=\phi\left(\frac{x-u}{l(u)}\right) \sqrt{J(x, u)} l(u)^{d / 2},
$$

such that $\phi_{u}$ is supported in $\{x:|x-u|<l(u)\}$. By definition, the function $l(u)$ is smooth and satisfies $0<l(u) \leq 1 / 2$ and $\|\nabla l\|_{\infty} \leq 1 / 2$. Therefore, according to [13], the functions $\phi_{u}$ satisfy (2.2) and (2.3) for all $u \in \mathbb{R}^{d}$.

To prove the upper bound in Proposition 2.1, put

$$
\gamma=\int_{\mathbb{R}^{d}} \phi_{u}\left(\phi_{u} H(b) \phi_{u}\right)_{-}^{0} \phi_{u} l(u)^{-d} d u .
$$


Obviously, $\gamma \geq 0$ holds and in view of (2.3) also $\gamma \leq 1$, hence, by a variant of the variational principle discussed in Appendix B,

$$
-\operatorname{Tr}(H(b))_{-} \leq \operatorname{Tr}(\gamma H(b))=-\int_{\mathbb{R}^{d}} \operatorname{Tr}\left(\phi_{u} H(b) \phi_{u}\right)_{-} l(u)^{-d} d u .
$$

To prove the lower bound we use the IMS-formula. For $\phi \in C_{0}^{\infty}\left(\mathbb{R}^{d}\right)$ and $v \in H^{1}(\Omega)$ we have

$$
\frac{1}{2} \nabla v \cdot \nabla\left(\phi^{2} \bar{v}\right)+\frac{1}{2} \nabla \bar{v} \cdot \nabla\left(\phi^{2} v\right)=|\nabla(\phi v)|^{2}-|\nabla \phi|^{2}|v|^{2}
$$

Combining this identity with the partition of unity (2.3) yields

$$
q_{b}[v]=\int_{\mathbb{R}^{d}}\left(q_{b}\left[\phi_{u} v\right]-\left(v, h^{2}\left(\nabla \phi_{u}\right)^{2} v\right)_{L^{2}(\Omega)}\right) l(u)^{-d} d u .
$$

Using (2.2) and (2.3) one can show [13], for every $x \in \mathbb{R}^{d}$,

$$
\int_{\mathbb{R}^{d}}\left(\nabla \phi_{u}\right)^{2}(x) l(u)^{-d} d u \leq C \int_{\mathbb{R}^{d}} \phi_{u}^{2}(x) l(u)^{-d-2} d u .
$$

We insert this into (5.1) and deduce

$$
\operatorname{Tr}(H(b))_{-} \leq \int_{\Omega^{*}} \operatorname{Tr}\left(\phi_{u}\left(H(b)-C h^{2} l(u)^{-2}\right) \phi_{u}\right)_{-} l(u)^{-d} d u
$$

where $\Omega^{*}=\left\{u \in \mathbb{R}^{d}: \operatorname{supp} \phi_{u} \cap \Omega \neq \emptyset\right\}$. For any $u \in \mathbb{R}$, let $\rho_{u}$ be another parameter $0<\rho_{u}<1$ and estimate

$$
\begin{aligned}
& \operatorname{Tr}\left(\phi_{u}\left(H(b)-C h^{2} l(u)^{-2}\right) \phi_{u}\right)_{-} \leq \operatorname{Tr}\left(\phi_{u} H(b) \phi_{u}\right)_{-} \\
& \quad+\operatorname{Tr}\left(\phi_{u}\left(\rho_{u} H(b)-C h^{2} l(u)^{-2}\right) \phi_{u}\right)_{-} .
\end{aligned}
$$

We now claim that choosing $\rho_{u}$ proportional to $h^{2} l(u)^{-2}$ yields

$$
\begin{aligned}
& \operatorname{Tr}\left(\phi_{u}\left(H(b)-C h^{2} l(u)^{-2}\right) \phi_{u}\right)_{-} \leq \operatorname{Tr}\left(\phi_{u} H(b) \phi_{u}\right)_{-} \\
& +C \frac{l(u)^{d-2}}{h^{d-2}}\left(1+\frac{\left(b_{m}\right)_{-}^{d+1} h}{l(u)}\right) .
\end{aligned}
$$


To see this, let us write $\tau_{u}=\rho_{u} /\left(\rho_{u}+C h^{2} l(u)^{-2}\right)$ and note that $\tau_{u}<1$ and $\operatorname{Tr}\left(\phi_{u}\left(\rho_{u} H(b)-C h^{2} l(u)^{-2}\right) \phi_{u}\right)_{-}=C h^{2} l(u)^{-2}\left(1-\tau_{u}\right)^{-1} \operatorname{Tr}\left(\phi_{u} \tilde{H}\left(\sqrt{\tau_{u}} b\right) \phi_{u}\right)_{-}$.

Here $\tilde{H}$ is generated by the same quadratic form as $H$ but with $h$ replaced by $\sqrt{\tau_{u}} h$. If supp $\phi_{u} \cap \partial \Omega \neq \emptyset$, we have $l_{0} / 4 \leq l(u) \leq l_{0} / \sqrt{3}$, see (2.10) and (2.11), and we can apply Corollary 4.3 to estimate

$$
\operatorname{Tr}\left(\phi_{u} \tilde{H}\left(\sqrt{\tau_{u}} b\right) \phi_{u}\right)_{-} \leq C l(u)^{d} h^{-d} \tau_{u}^{-d / 2}\left(1+\left(b_{m}\right)_{-}^{d+1} h l(u)^{-1}\right)
$$

With our choice of $\rho_{u}$ proportional to $h^{2} l(u)^{-2}$ we find that $\tau_{u}$ is order one and (5.3) follows. If $\phi_{u} \in C_{0}^{\infty}(\Omega)$ we can argue similarly by using the lower bound in Proposition 2.2 and get

$$
\operatorname{Tr}\left(\phi_{u}\left(H(b)-C h^{2} l(u)^{-2}\right) \phi_{u}\right)_{-} \leq \operatorname{Tr}\left(\phi_{u} H(b) \phi_{u}\right)_{-}+C \frac{l(u)^{d-2}}{h^{d-2}}
$$

Finally, we insert (5.3) and (5.4) into (5.2) and arrive at

$$
\begin{aligned}
\operatorname{Tr}(H(b))_{-} \leq & \int_{\Omega^{*}} \operatorname{Tr}\left(\phi_{u} H(b) \phi_{u}\right)_{-} l(u)^{-d} d u+C h^{-d+2} \int_{\Omega \backslash U} l(u)^{-2} d u \\
& +C h^{-d+2} \int_{U}\left(l(u)^{-2}+\left(b_{m}\right)_{-}^{d+1} h l(u)^{-3}\right) d u,
\end{aligned}
$$

where $U=\left\{u \in \mathbb{R}^{d}: \partial \Omega \cap B_{u} \neq \emptyset\right\}$. Thus the claim of Proposition 2.1 follows from (2.15) and (2.16).

Open Access This article is distributed under the terms of the Creative Commons Attribution License which permits any use, distribution, and reproduction in any medium, provided the original author(s) and the source are credited.

\section{Appendix A: A geometric lemma}

In the proofs of Theorem 1.1 and Theorem 1.3 we used the following estimate.

Lemma 6.1 For every bounded domain $\Omega \subset \mathbb{R}^{d}$ with $\partial \Omega \in C^{1}$ there is a constant $C$ with the following property. For every $0<l_{0} \leq 1$ and $u \in \mathbb{R}^{d}$ let $l(u)$ be defined as in (2.1) by

$$
l(u)=\frac{1}{2}\left(1+\left(\operatorname{dist}\left(u, \mathbb{R}^{d} \backslash \Omega\right)^{2}+l_{0}^{2}\right)^{-1 / 2}\right)^{-1}
$$


Then for any relatively open $N \subset \partial \Omega$ the set

$$
U^{*}=\left\{u \in \mathbb{R}^{d}: \operatorname{dist}(u, \partial \Omega)<l(u) \wedge \operatorname{dist}(u, \partial \Omega \backslash N)>l(u)\right\}
$$

satisfies

$$
\limsup _{l_{0} \downarrow 0} \frac{1}{l_{0}}\left|U^{*}\right|_{d} \leq C \sigma(N) .
$$

Here $|\cdot|_{d}$ denotes the d-dimensional Lebesgue measure on $\mathbb{R}^{d}$ and $\sigma(\cdot)$ denotes the $d-1$-dimensional surface measure on $\partial \Omega$.

Proof We split $U^{*}$ into two parts $U_{i}^{*}=U^{*} \cap \Omega$ and $U_{o}^{*}=U^{*} \cap \mathbb{R}^{d} \backslash \Omega$ and we prove the assertion separately for each of them. We begin with $U_{i}^{*}$. Note that for $u \in \Omega$ we have $\operatorname{dist}\left(u, \mathbb{R}^{d} \backslash \Omega\right)=\operatorname{dist}(u, \partial \Omega)$. We first argue that there is a constant $L_{l_{0}}$ such that

$$
U_{i}^{*}=\left\{u \in \Omega: \operatorname{dist}(u, \partial \Omega)<L_{l_{0}} \wedge \operatorname{dist}(u, \partial \Omega \backslash N)>l(u)\right\}
$$

and such that $l_{0} / 4 \leq L_{l_{0}} \leq l_{0} / \sqrt{3}$.

To prove (6.1) let us consider the function

$$
F_{l_{0}}(x)=\frac{1}{2}\left(1+\left(x^{2}+l_{0}^{2}\right)^{-1 / 2}\right)^{-1}-x, \quad x \geq 0 .
$$

This function is continuously differentiable and satisfies $F_{l_{0}}(0)=l_{0} /\left(2\left(l_{0}+1\right)\right)>0$, $F_{l_{0}}(x) \leq 0$ for $x \geq 1 / 2$, and

$$
F_{l_{0}}^{\prime}(x)=\frac{x}{2}\left(x^{2}+l_{0}^{2}\right)^{-1 / 2}\left(1+\left(x^{2}+l_{0}^{2}\right)^{1 / 2}\right)^{-2}-1 \leq-\frac{1}{2}
$$

for all $x \geq 0$. Hence, there is a unique $L_{l_{0}} \in(0,1 / 2]$ with $F_{l_{0}}\left(L_{l_{0}}\right)=0$. Moreover, since $F_{l_{0}}\left(l_{0} / 4\right)<0<F_{l_{0}}\left(l_{0} / \sqrt{3}\right)$, we have $l_{0} / 4<L_{l_{0}}<l_{0} / \sqrt{3}$.

By definition, all $u \in \Omega$ with $\operatorname{dist}(u, \partial \Omega)=L_{l_{0}}$ satisfy $F_{l_{0}}(\operatorname{dist}(u, \partial \Omega))=0$, thus $l(u)=\operatorname{dist}(u, \partial \Omega)=L_{l_{0}}$. The fact that $F_{l_{0}}$ is decreasing shows that the inequality $\operatorname{dist}(u, \partial \Omega)<L_{l_{0}}$ implies $F_{l_{0}}(\operatorname{dist}(u, \partial \Omega))>0$, thus $\operatorname{dist}(u, \partial \Omega)<l(u)$. Similarly, the inequality $\operatorname{dist}(u, \partial \Omega)<l(u) \operatorname{implies} \operatorname{dist}(u, \partial \Omega)<L_{l_{0}}$. This proves (6.1).

Our next step is to fix an $0<\epsilon<1$ and to decompose $U_{i}^{*}=U_{>}^{*} \cup U_{\epsilon}^{*}$ with

$$
\begin{aligned}
& U_{>}^{*}=\left\{u \in \Omega: \operatorname{dist}(u, \partial \Omega)<(1-\epsilon) L_{l_{0}} \wedge \operatorname{dist}(u, \partial \Omega \backslash N)>l(u)\right\} \\
& U_{\epsilon}^{*}=\left\{u \in \Omega:(1-\epsilon) L \leq \operatorname{dist}(u, \partial \Omega)<L_{l_{0}} \wedge \operatorname{dist}(u, \partial \Omega \backslash N)>l(u)\right\} .
\end{aligned}
$$

Thus,

$$
\left|U_{i}^{*}\right|_{d} \leq\left|U_{>}^{*}\right|_{d}+\left|U_{\epsilon}^{*}\right|_{d}
$$


The second term on the right side can easily be bounded,

$$
\left|U_{\epsilon}^{*}\right|_{d} \leq|\{u \in \Omega:(1-\epsilon) L \leq \operatorname{dist}(u, \partial \Omega)<L\}|_{d} \leq \int_{(1-\epsilon) L_{l_{0}}}^{L_{l_{0}}} \sigma\left(\partial \Omega_{t}\right) d t \leq C l_{0} \epsilon
$$

Here we wrote $\partial \Omega_{t}=\{u \in \Omega: \operatorname{dist}(u, \partial \Omega)=t\}$ and used the facts that $\sigma\left(\partial \Omega_{t}\right)$ is uniformly bounded and that $L_{l_{0}} \leq l_{0} / \sqrt{3}$.

After these steps we have reduced the lemma to proving that

$$
\underset{l_{0} \downarrow 0}{\limsup } \frac{1}{l_{0}}\left|U_{>}^{*}\right|_{d} \leq C \sigma(N)
$$

with a constant $C$ independent of $\epsilon$. To do so we start from the representation

$$
\left|U_{>}^{*}\right|_{d}=\int_{0}^{(1-\epsilon) L_{l_{0}}} \sigma\left(U_{t}^{*}\right) d t
$$

where

$U_{t}^{*}=\{u \in \Omega: \operatorname{dist}(u, \partial \Omega)=t \wedge \operatorname{dist}(u, \partial \Omega \backslash N)>l(u)\}, \quad 0 \leq t<(1-\epsilon) L_{l_{0}}$.

Recall that every $u \in U^{*}$ and, in particular, every $u \in U_{>}^{*}$ satisfies $\operatorname{dist}(u, \partial \Omega)<$ $l(u)$. We now claim that for every $0<\epsilon<1$ and every $0<l_{0} \leq 1$ there is an $r>0$ such that every $u \in U_{>}^{*}$ satisfies

$$
l(u)>\operatorname{dist}(u, \partial \Omega)+r .
$$

This follows again from the monotonicity and continuity of the function $F_{l_{0}}$. Indeed, we can set $r=F_{l_{0}}\left((1-\epsilon) L_{l_{0}}\right)$.

We consider the set

$$
\tilde{N}:=\bigcup_{0<t<(1-\epsilon) L_{l_{0}}} \bigcup_{u \in U_{t}^{*}} \bigcup_{x \in \partial \Omega,|x-u|=t}\left\{y \in \mathbb{R}^{d}:|y-x|<r\right\} \cap \partial \Omega
$$

and show that

$$
\tilde{N} \subset N
$$

and

$$
\sigma(\partial \tilde{N})=0
$$


To prove (6.4) let $0<t<(1-\epsilon) L_{l_{0}}, x, y \in \partial \Omega$ with $|x-y|<r$ and $u \in U_{t}^{*}$ with $|x-u|=t$. Then

$$
|y-u| \leq|y-x|+|x-u|<r+\operatorname{dist}(u, \partial \Omega)<l(u) .
$$

Since $\operatorname{dist}(u, \partial \Omega \backslash N)>l(u)$ by the definition of $U_{t}^{*}$, we infer that $y \in N$. This proves (6.4).

To prove (6.5) we note that $\tilde{N}$ satisfies the following uniform interior ball condition. For each $y \in \partial \tilde{N}$ there is an open ball $B \subset \mathbb{R}^{d}$ of radius $r$ such that $y \in \partial B$ and $B \cap \partial \Omega \subset \tilde{N}$. In order to prove (6.5) we introduce local coordinates similarly as in Sect. 4. In this way we are reduced to the situation where $\tilde{N}$ is a subset of $\mathbb{R}^{d-1}$ satisfying a uniform interior ball condition (with a possibly smaller radius). The claim (6.5) follows from Lemma 6.2 below.

The definition of $\tilde{N}$ easily implies that

$$
U_{t}^{*} \subset \tilde{U}_{t}^{*}:=\{u \in \Omega: \operatorname{dist}(u, \partial \Omega)=t \wedge \operatorname{dist}(u, \tilde{N})=t\}
$$

for all $0 \leq t<(1-\epsilon) L_{l_{0}}$. Moreover, we can estimate with a constant depending only on $\Omega$

$$
\begin{aligned}
\sigma\left(\tilde{U}_{t}^{*}\right) & \leq C(\sigma(\tilde{N})+\sigma(\{x \in \partial \Omega \backslash \tilde{N}: \operatorname{dist}(x, \tilde{N})<t\})) \\
& \leq C\left(\sigma(N)+\sigma\left(\left\{x \in \partial \Omega \backslash \tilde{N}: \operatorname{dist}(x, \tilde{N})<l_{0}\right\}\right)\right) .
\end{aligned}
$$

The second bound used (6.4) as well as $(1-\epsilon) L_{l_{0}} \leq(1-\epsilon) l_{0} / \sqrt{3} \leq l_{0}$. Thus, from (6.3),

$$
\left|U_{>}^{*}\right|_{d} \leq C l_{0}\left(\sigma(N)+\sigma\left(\left\{x \in \partial \Omega \backslash \tilde{N}: \operatorname{dist}(x, \tilde{N})<l_{0}\right\}\right)\right)
$$

Therefore, in order to prove (6.2), it remains to estimate

$$
\sigma\left(\left\{x \in \partial \Omega \backslash \tilde{N}: \operatorname{dist}(x, \tilde{N})<l_{0}\right\}\right)=\int_{\partial \Omega} \chi_{l_{0}}(y) d \sigma(y),
$$

where $\chi_{l_{0}}$ denotes the characteristic function of $\left\{x \in \partial \Omega \backslash \tilde{N}: \operatorname{dist}(x, \tilde{N})<l_{0}\right\}$. We note that $\lim _{l_{0} \downarrow 0} \chi_{l_{0}}=\chi_{\partial \tilde{N}}$ pointwise. Thus, the dominated convergence theorem and (6.5) imply that

$$
\lim _{l_{0} \downarrow 0} \sigma\left(\left\{x \in \partial \Omega \backslash \tilde{N}: \operatorname{dist}(x, \tilde{N})<l_{0}\right\}\right)=0 .
$$

This completes the proof of (6.2).

For $U_{o}^{*}$ we get an analogous bound by following the same strategy. In this case the estimates are somewhat simpler since, for $u \in \mathbb{R}^{d} \backslash \Omega$, we have $l(u) \equiv \frac{1}{2} l_{0} /\left(l_{0}+1\right)$ and this plays the role of $L_{l_{0}}$. 
Lemma 6.2 Let $A \subset \mathbb{R}^{n}$ be bounded. Assume that there is $\rho>0$ such that for each $x \in \partial A$ there is a ball $B \subset \mathbb{R}^{n}$ of radius $\rho$ with $x \in \partial B$ and $B \subset A$. Then $|\partial A|_{n}=0$.

Proof Let $\delta>0$ be a constant to be specified later and put $l_{m}=\delta \rho 5^{-m}$ for $m \geq 0$. We denote by $\mathcal{Q}_{m}$ the collection of open cubes of side length $l_{m}$ centered at points in $\left(l_{m} \mathbb{Z}\right)^{n}$. Let $\mathcal{C}_{m}$ be the collection of those cubes in $\mathcal{Q}_{m}$ that intersect both $A$ and $\mathbb{R}^{n} \backslash A$. Since $A$ is bounded, $v_{m}:=\# \mathcal{C}_{m}$ is finite. We claim that for all sufficiently small $\delta>0$ there is a constant $M<5^{n}$ such that for all $m \geq 1$

$$
v_{m} \leq M v_{m-1} .
$$

Deferring the proof of this bound for the moment we now explain why it implies the lemma. First, we iterate (6.6) to learn that $v_{m} \leq M^{m} v_{0}$. Thus, since $\partial A \subset \bigcup_{Q \in \mathcal{C}_{m}} \bar{Q}$ for any $m$, we conclude that

$$
|\partial A|_{n} \leq \sum_{Q \in \mathcal{C}_{m}}|Q|_{n}=l_{m}^{n} v_{m} \leq \delta^{n} \rho^{n}\left(5^{-n} M\right)^{m} v_{0} \rightarrow 0 \quad \text { as } m \rightarrow \infty .
$$

This proves $|\partial A|_{n}=0$ and we are left with showing (6.6).

To do so, we fix $m \geq 1$ and an arbitrary cube $Q \subset \mathcal{C}_{m-1}$. When passing from $m-1$ to $m$, this cube is subdivided into $5^{n}$ cubes in $\mathcal{Q}_{m}$. We shall show that if $\delta>0$ is sufficiently small then at least one of these cubes of side length $l_{m}$ does not belong to $\mathcal{C}_{m}$ (i.e., does not intersect both $A$ and $\mathbb{R}^{n} \backslash A$ ). This will imply (6.6) with $M=5^{n}-1$.

Consider the cube $Q^{\prime} \in \mathcal{Q}_{m}$ in the center of $Q$. If this cube does not belong to $\mathcal{C}_{m}$ we are done. Thus, we may assume that $Q^{\prime}$ intersects both $A$ and $\mathbb{R}^{n} \backslash A$. Because of our assumption on $\partial A$ there is an open ball $B$ of radius $\rho$ such that $B \subset A$ and $\partial B \cap Q^{\prime} \neq \varnothing$. We now make use of the following

Claim. There is a constant $C_{n}>0$ such that if $B \subset \mathbb{R}^{n}$ is an open ball of radius $r \geq C_{n}$ with $\bar{B} \cap Q \neq \emptyset$, where $Q=(-1 / 2,1 / 2)^{n}$, then $\gamma+Q \subset B$ for some $\gamma \in \mathbb{Z}^{n}$ with $|\gamma|_{\infty} \leq 2$

Indeed, one can take $C_{n}=\max \{\sqrt{n}, n / 2\}$. The proof of this claim uses only elementary geometric facts and is omitted.

By a rescaled version of the claim we infer that, under the assumption that $\rho \geq C_{n} l_{m}$, there is a cube in $\mathcal{Q}_{m}$ which is contained in $B$ and whose center is at most an $\infty$-distance $2 l_{m}$ away from that of $Q^{\prime}$. Since $Q^{\prime}$ lies in the center of $Q$ this cube is also contained in $Q$. Moreover, since it is contained in $B$, it is also contained in $A$ and, therefore, does not belong to $\mathcal{C}_{m}$.

Finally, we argue that for all $\delta>0$ small enough the assumption $\rho \geq C_{n} l_{m}$ is satisfied for all $m \geq 1$. Indeed, this assumption is equivalent to $1 \geq C_{n} \delta 5^{-m}$, which holds uniformly in $m \geq 1$ provided we choose $\delta \leq 5 C_{n}^{-1}$. This completes the proof.

\section{Appendix B: A variant of the variational principle and a sharp bound on $\operatorname{Tr}\left(-\boldsymbol{\Delta}_{c}-\boldsymbol{\Lambda}\right)_{-}$}

Here we mention the following extension of the variational principle that we used in the proof of Proposition 3.1. 
Let $(M, \mu)$ be a measure space and let $\left(f_{\alpha}\right)_{\alpha \in M}$ be a measurable family of functions in a separable Hilbert space $\mathcal{G}$, such that

$$
\int_{M}\left|\left(\psi, f_{\alpha}\right)\right|^{2} d \mu(\alpha) \leq\|\psi\|^{2}
$$

for all $\psi \in \mathcal{G}$. Assume that $A$ is a self-adjoint, lower semibounded operator in $\mathcal{G}$ with quadratic form $a$ such that

$$
f_{\alpha} \in \operatorname{dom}[a]
$$

for all $\alpha \in M$.

Let the operator $\gamma$ in $\mathcal{G}$ be given by $\gamma \psi=\int_{M}\left(f_{\alpha}, \psi\right) f_{\alpha} d \mu(\alpha)$. Then $\gamma$ satisfies $0 \leq \gamma \leq 1$. Let us introduce the notation

$$
\operatorname{Tr} A \gamma=\int_{M} a\left[f_{\alpha}\right] d \mu(\alpha)
$$

Then we have

$$
-\operatorname{Tr} A_{-} \leq \operatorname{Tr} A \gamma
$$

provided $\int_{M} a\left[f_{\alpha}\right]_{-} d \mu(\alpha)<\infty$.

Let us illustrate these notions by adding the following sharp estimate, a simple form of the upper in Proposition 3.1, which is based on a method introduced in [10]. Here we only assume that the boundary of $\Omega \subset \mathbb{R}^{d}$ is Lipschitz continuous and that $-\Delta_{c}$ is generated by the quadratic form given in (1.1).

Proposition 7.1 For $\phi \in C_{0}^{1}\left(\mathbb{R}^{d}\right)$ and $\Lambda>0$

$$
\begin{aligned}
\operatorname{Tr}\left(\phi\left(-\Delta_{c}-\Lambda\right) \phi\right)_{-} \geq & L_{d}^{(1)} \Lambda^{1+d / 2} \int_{\Omega}|\phi(x)|^{2} d x \\
& \quad-\frac{\omega_{d}}{(2 \pi)^{d}} \Lambda^{d / 2}\left(\int_{\partial \Omega} c(x)|\phi(x)|^{2} d \sigma(x)+\int_{\Omega}|\nabla \phi|^{2} d x\right) .
\end{aligned}
$$

Proof To adopt the notation introduced above, we set $\mathcal{G}=L^{2}(\Omega), M=\left\{\xi \in \mathbb{R}^{d}\right.$ : $\left.|\xi|^{2} \leq \Lambda\right\}$ and $\mu$ to be Lebesgue measure. If we choose $f_{\xi}(x)=(2 \pi)^{-d / 2} e^{i x \cdot \xi}$ then (7.1) and (7.2) are satisfied and the claim follows from (7.3).

If we choose $\phi \equiv 1$ on $\Omega$ we get

$$
\operatorname{Tr}\left(-\Delta_{c}-\Lambda\right)_{-} \geq L_{d}^{(1)}|\Omega| \Lambda^{1+d / 2}-\frac{\omega_{d}}{(2 \pi)^{d}} \int_{\partial \Omega} c(x) d \sigma(x) \Lambda^{d / 2} .
$$


This generalizes the bound proved in [10] for the case of Neumann boundary conditions.

\section{References}

1. Abramowitz, M., Stegun, I.A.: Handbook of Mathematical Functions with Formulas, Graphs, and Mathematical Tables. National Bureau of Standards Applied Mathematics Series, vol. 55 (1964)

2. Birman, M.Š., Solomjak, M.Z.: Quantitative analysis in Sobolev imbedding theorems and applications to spectral theory. American Mathematical Society Translations, Series 2, vol. 114. American Mathematical Society, Providence (1980)

3. Branson, T.P., Gilkey, P.B.: The asymptotics of the Laplacian on a manifold with boundary. Commun. Partial Differ. Equ. 15(2), 245-272 (1990)

4. Frank, R.L., Geisinger, L.: Two-term spectral asymptotics of the Dirichlet Laplacian on a bounded domain. In: Exner, P. (ed.) Mathematical Results in Quantum Physics: Proceedings of the Qmath11 Conference, pp. 138-147. World Scientific Publishing Company, Singapore (2011)

5. Frank, R.L., Geisinger, L.: Refined semiclassical asymptotics for fractional powers of the Laplace operator. Preprint (2011). arXiv:1105.5181

6. Hörmander, L.: The Analysis of Linear Partial Differential Operators, vol. 4. Springer, Berlin (1985)

7. Ivrii, V.J.: The second term of the spectral asymptotics for the Laplace-Beltrami operator on manifolds with boundary. Funktsional. Anal. i Prilozhen. 14(2), 25-34 (1980)

8. Ivrii, V.J.: The second term of the spectral asymptotics for the Laplace-Beltrami operator on manifolds with boundary and for elliptic operators acting in vector bundles. Soviet Math. Dokl. 20(1), 1300-1302 (1980)

9. Ivrii, V.J.: Microlocal analysis and precise spectral asymptotics. In: Springer Monographs in Mathematics. Springer, Berlin (1998)

10. Kröger, P.: Upper bounds for the Neumann eigenvalues on a bounded domain in Euclidean space. J. Funct. Anal. 106(2), 353-357 (1992)

11. McKean, H.P. Jr., Singer, I.M.: Curvature and the eigenvalues of the Laplacian. J. Differ. Geom. 1(1), 43-69 (1967)

12. Pleijel, Å.: A study of certain Green's functions with applications in the theory of vibrating membranes. Ark. Mat. 2, 553-569 (1954)

13. Solovej, J.P., Spitzer, W.L.: A new coherent states approach to semiclassics which gives Scott's correction. Commun. Math. Phys. 241(2-3), 383-420 (2003)

14. Safarov, Y., Vassiliev, D.: The asymptotic distribution of eigenvalues of partial differential operators. In: Translations of Mathematical Monographs, vol. 155. American Mathematical Society, Providence (1997)

15. Weyl, H.: Das asymptotische Verteilungsgesetz der Eigenwerte linearer partieller Differentialgleichungen (mit einer Anwendung auf die Theorie der Hohlraumstrahlung). Math. Ann. 71(4), 441-479 (1912) 